J. Math. Biology (1982) 15: I-36

\title{
The Stabilizing Effect of a Random Environment
}

\author{
Peter L. Chesson* \\ Department of Biological Sciences and Marine Science Institute, University of California, Santa \\ Barbara, CA 93106, USA
}

\begin{abstract}
It is shown that the lottery competition model permits coexistence in a stochastic environment, but not in a constant environment. Conditions for coexistence and competitive exclusion are determined. Analysis of these conditions shows that the essential requirements for coexistence are overlapping generations and fluctuating birth rates which ensure that each species has periods when it is increasing. It is found that a species may persist provided only that it is favored sufficiently by the environment during favorable periods independently of the extent to which the other species is favored during its favorable periods.

Coexistence is defined in terms of the stochastic boundedness criterion for species persistence. Using the lottery model as an example this criterion is justified and compared with other persistence criteria. Properties of the stationary distribution of population density are determined for an interesting limiting case of the lottery model and these are related to stochastic boundedness. An attempt is then made to relate stochastic boundedness for infinite population models to the behavior of finite population models.
\end{abstract}

Key words: Stochastic competition models - Stochastic stability - Stochastic boundedness

\section{Introduction}

Theoretical ecologists have tended to regard a stochastic environment as inimical to species coexistence. Indeed, the usual stability analysis of deterministic models is often conducted from this viewpoint. Common reasoning is that coexistence occurs only if the model system can withstand environmental fluctuations, and for this, it is argued, there must be an invariant set, such as an equilibrium point or cycle, with a large domain of attraction. Also there must be rapid return to this invariant set following perturbation. Detailed discussions of these ideas are given by Lewontin (1969), Holling (1973), May (1974), Leigh (1975), Beddington et al. (1976), and Goh $(1975,1976)$.

* Present address: Department of Zoology, The Ohio State University, 1735 Neil Avenue, Columbus, Ohio 43210, USA 
The point of view represented above undoubtedly has some validity, but environmental fluctuations are not always bad. For example, it is now well known that some kinds of deterministic fluctuations in the environment may permit coexistence in models where a constant environment does not (Stewart and Levin, 1973; Koch, 1974; Armstrong and McGehee, 1976; Levins, 1979; Cushing, 1980; de Mottoni and Schiaffino, 1981). That a stochastic environment can promote coexistence was shown by Chesson and Warner (1981) in a model of competition for space. Chesson and Warner demonstrate heuristically that stochastic fluctuations in the environment can provide opportunities for a species to increase against a generally superior competitor. Coexistence is then possible provided the inferior species does not decline too rapidly during unfavorable periods at low population densities. Such coexistence relies on environmental fluctuations, indeed coexistence is more likely with stronger fluctuations.

The case of a stochastic environment studied by Chesson and Warner is an important one because environmental variability commonly has a highly stochastic component and it is mainly this stochastic component that people have in mind when analyzing the stability of a deterministic model.

This paper provides the mathematical demonstration of Chesson and Warner's heuristically justified results. Since the analysis seeks situations in which species coexistence occurs, it necessarily becomes involved in the important question of how to define species persistence in a stochastic model. There are many ideas on this (May, 1974; Botkin and Sobel, 1975; Ludwig, 1976; Turelli, 1978a, 1981 ; Chesson, 1978). The analysis here illustrates and justifies one particular method of defining species persistence, the stochastic boundedness criterion (Chesson, 1978) which can be stated as follows:

A species with population size $N(t)$ at time $t$ is said to persist in the sense of stochastic boundedness if

$$
\inf _{\varepsilon>0} \sup _{i} \mathscr{P}(N(t)<\varepsilon)=0
$$

This will be so, for example, if $N(t)$ converges in distribution to a positive random variable as $t \rightarrow \infty$, but the idea of stochastic boundedness is more general than this for (1) does not imply convergence in distribution. The criterion is best understood in terms of the quantiles $\zeta_{p}(t)$ of the probability distribution of $N(t)$, which are defined by the equation $\mathscr{P}\left(N(t) \leqslant \zeta_{p}(t)\right)=p$, i.e. $\zeta_{p}(t)$ cuts off the lower proportion $p$ of the probability distribution of $N(t)$. Equation (1) is satisfied if and only if $\zeta_{p}(t)$ is bounded below by some positive number $\xi_{p}$ independent of $t$, for every $p$. Thus stochastic boundedness generalizes the deterministic notion that a species persists if $N(t)$ is bounded below by a positive constant independent of $t$. To take this analogy further, let $N^{*}$ be the positive random variable with quantiles $\xi_{p}$, then $N(t)$ is stochastically larger than $N^{*}$ for all $t$, i.e. $\mathscr{P}\left(N^{*} \leqslant x\right) \geqslant \mathscr{P}(N(t) \leqslant x)$ for all $t$ and $x$. As a short hand, a population that persists in the sense of stochastic boundedness will be said to be s.b. persistent.

The justification of the stochastic boundedness criterion is discussed here in detail using the lottery model as an example. However the first sections of the paper analyze the lottery model to determine the conditions under which the species do coexist in the sense that both species are s.b. persistent; and these conditions are 
analyzed to determine the kinds of environmental fluctuations that are favorable to coexistence.

\section{The Population Model}

Chesson and Warner's lottery model is based on a description by Sale (1977) of the possible workings of a coral reef fish community. Consider just two species and assume that the adults require a territory or "home" (an area held to the exclusion of others) in order to reproduce. The individuals compete for space to establish homes and it is assumed that this space is always in short supply. Thus if $P_{i}(t)$ is the proportion of space occupied by species $i$, then $P_{1}(t)+P_{2}(t)=1$.

During the time interval $(t, t+1], \delta_{i}(t)$ of the adults of species $i$ die and so the proportion of space becoming available during this time interval is $\delta_{1}(t) P_{1}(t)$ $+\delta_{2}(t) P_{2}(t)$. Juveniles settle in this space and become adults.

The number of juveniles of species $i$ seeking homes during $(t, t+1]$ is $\beta_{i}(t) P_{i}(t)$ and it is assumed that the proportion of the available space taken up by species $i$ is equal to

$$
\frac{c_{i} \beta_{i}(t) P_{i}(t)}{c_{1} \beta_{1}(t) P_{1}(t)+c_{2} \beta_{2}(t) P_{2}(t)} .
$$

Formula (2) embodies the lottery aspect of the model. It represents an allocation of space that is random within species but possibly biased between species. If allocation of space is random between species then $c_{1}=c_{2}$. In general the parameter $c_{i}$ may be interpreted as proportional to the per capita rate of discovery of available space by species $i$ and is a measure of the competitive ability of species $i$.

Putting all of this together we obtain the following equations governing population change:

$$
P_{1}(t+1)=\left[1-\delta_{1}(t)\right] P_{1}(t)+\left[\delta_{1}(t) P_{1}(t)+\delta_{2}(t) P_{2}(t)\right] \frac{\beta_{1}^{*}(t) P_{1}(t)}{\beta_{1}^{*}(t) P_{1}(t)+\beta_{2}^{*}(t) P_{2}(t)}
$$

where $\beta_{i}^{*}(t)=c_{i} \beta_{i}(t)$ the "birth-competition" parameter. There is an analogous equation for species 2 but since $P_{1}(t)+P_{2}(t)=1$ equation (3) completely specifies the system. In this equation environmental variability is specified by variation in $\beta_{i}^{*}(t)$ and $\delta_{i}(t)$, both of which are assumed strictly positive. The environment process is $\mathscr{E}=\{\mathscr{E}(t), t=0,1, \ldots\}$ where $\mathscr{E}(t)=\left(\beta_{1}^{*}(t), \delta_{1}(t), \beta_{2}^{*}(t), \delta_{2}(t)\right)$.

In writing down equation (3) a number of implicit assumptions have been introduced. Like most deterministic models, and stochastic models with random environment, it treats population size as a continuous variable and it makes no allowances for stochasticity at the level of the individual animal (within-individual variability, Chesson, 1978). As such it effectively assumes an infinite population spread homogeneously over an infinite area (Chesson, 1981). The assumption of homogeneity is partly justified if the juvenile organisms disperse widely as Sale (1977) claims is true for some coral reef fishes. The effect of the infinite population is discussed below in the section on finite populations and stochastic boundedness. 
To analyze the model, equation (3) can be rearranged to give

$$
P_{1}(t+1)=P_{1}(t)\left\{1+\left[1-P_{1}(t)\right] \frac{\beta_{1}^{*}(t) \delta_{2}(t)-\beta_{2}^{*}(t) \delta_{1}(t)}{\beta_{1}^{*}(t) P_{1}(t)+\beta_{2}^{*}(t) P_{2}(t)}\right\} .
$$

It is clear from (4) that $P_{1}(t+1)$ is greater than $P_{1}(t)$ or less than $P_{1}(t)$ depending on whether $\beta_{1}^{*}(t) \delta_{2}(t)-\beta_{2}^{*}(t) \delta_{1}(t)$ is positive or negative. Define $\rho=$ $\beta_{1}^{*}(t) \delta_{2}(t) / \beta_{2}^{*}(t) \delta_{1}(t)$ and consider the case of a constant environment (where $\beta_{i}^{*}(t)$ and $\delta_{i}(t)$ are independent of $\left.t, i=1,2\right)$. It is clear that $P_{1}(t) \rightarrow 0$ or 1 depending on whether $\rho<1$ or $\rho>1$. For $\rho=1$ all possible values of $P_{1}(t)$ are neutral equilibria and so $P_{1}(t)$ remains constant for all time. Unless $\rho=1$ a constant environment does not permit coexistence in this model. In the standard interpretation of deterministic models (e.g. May, 1974) the neutral stability of the case $\rho=1$ would not be regarded as giving coexistence because there is no deterministic stabilizing tendency and therefore environmental variability should lead to extinction of one species. However when environmental variability is included in the model we do not always find this; indeed, there are broad ranges of parameter values giving a strong coexistence even though the deterministic analogue has neutral stability (see below).

The stochastic versions of this model behave differently depending on whether generations are overlapping $\left(\delta_{i}(t)<1\right)$ or nonoverlapping $\left(\delta_{i}(t) \equiv 1\right)$. When generations are nonoverlapping equation (3) can be written in the simple linear form

$$
P_{1}(t+1) / P_{2}(t+1)=\rho(t)\left[P_{1}(t) / P_{2}(t)\right]
$$

where $\rho(t)=\beta_{1}^{*}(t) / \beta_{2}^{*}(t)$. Thus the behavior of $P_{1}(t)$ depends on the behavior of the product $\prod_{j=0}^{t} \rho(j)$. If the environment process $\mathscr{E}$ is stationary and ergodic then $P_{1}(t) \stackrel{\text { a.s. }}{\rightarrow} 0$ or $P_{1}(t) \stackrel{\text { a.s. }}{\rightarrow} 1$ depending on whether $E \log \rho$ is positive or negative, or equivalently depending on whether the geometric mean of $\rho$ is greater than 1 or less than 1 . Thus when $E \log \rho \neq 0$ we obtain results analogous to those for a constant environment.

When $E \log \rho=0$ the situation is not so clear. Assume that $\log \prod_{j=0}^{t} \rho(j)$ is asymptotically normal with mean 0 and variance $\sigma^{2} t$, as will be the case if the environment satisfies suitable moment and asymptotic independence conditions (Ibragimov, 1975; Heyde, 1974), then asymptotically $\log P_{1}(t) / P_{2}(t)$ has this same distribution which means that $P_{1}(t)$ converges in distribution to a random variable $P$ such that $\mathscr{P}(P=0)=\mathscr{P}(P=1)=\frac{1}{2}$. This means that neither species is s.b. persistent. However it is also clear that $\mathscr{P}\left(P_{i}(t) \rightarrow 0\right)=0, i=1,2$, so that with probability 1 neither species density converges to 0 . This suggests that both species persist, indeed having probability 0 of converging to 0 is highly intuitive as a criterion for persistence; but it is argued below, in the section on finite models and stochastic boundedness, that the stochastic boundedness criterion does give the correct interpretation here, and this particular situation should be regarded as resulting in extinction of one of the two species. Thus we conclude that in no case does the stochastic nonoverlapping generations model lead to species coexistence. However we shall see in the next section that different conclusions are obtained when generations are overlapping. 


\section{Boundary Analysis of the Stochastic Model with Overlapping Generations}

The analysis of the stochastic model with overlapping generations is divided into three parts. In this section we find criteria indicating whether $P_{i}(t) \rightarrow 0$ or $P_{i}(t) \rightarrow 0$; in section 4 we study the kinds of environmental variability for which these criteria are satisfied; and in section 5 we show that both species are s.b. persistent, i.e. coexistence occurs, for most of the cases where we have found $\mathscr{P}\left(P_{i}(t) \rightarrow 0\right)=0$, $i=1,2$.

We assume throughout this section that $0<\delta_{i}(t)<1$ and $\beta_{i}^{*}(t)>0$. Moreover we shall assume that $\mathscr{E}(0), \mathscr{E}(1), \ldots$ are i.i.d. although the proof of Theorem 3.1, dealing with cases of persistence, only requires a law of large numbers to hold.

To begin the analysis suppose that $P_{1}(t)$ is small. From equation (4) we have the approximation

$$
P_{1}(t+1) \simeq P_{1}(t)\left[1+\delta_{1}(\rho-1)\right]
$$

where $\rho=\beta_{1}^{*}(t) \delta_{2}(t) / \beta_{2}^{*}(t) \delta_{1}(t)$, and $t$ is suppressed in $\delta_{i}$ and $\rho$ for notational simplicity. From (6) we see that

$$
\Delta_{1}=E \log \left[1+\delta_{1}(\rho-1)\right]
$$

is an approximate average instantaneous growth rate for species 1 when $P_{1}(t)$ is small. The corresponding quantity for species 2 is

$$
\Delta_{2}=E \log \left[1+\delta_{2}\left(\rho^{-1}-1\right)\right] \text {. }
$$

We shall refer to $\Delta_{1}$ and $\Delta_{2}$ as the boundary growth rates. The usual invasibility analysis (Turelli, 1978b) now holds that $P_{i}(t)$ will tend to increase when small if $\Delta_{i}>0$. Indeed we show that this is so here and that also $\mathscr{P}\left(P_{i}(t) \rightarrow 0\right)=0$ whenever $\Delta_{i}>0$.

First of all we obtain a lower bound, independent of $P_{1}(t)$, to the mean instantaneous growth rate. We define $\Gamma\left(P_{1}(t)\right)$ to be the finite growth rate of species 1, i.e. $P_{1}(t+1) / P_{1}(t)=1+\Gamma\left(P_{1}(t)\right)$ and $\Gamma$ is given explicitly as

$$
\Gamma(p)=(1-p) \frac{\beta_{1}^{*}(t) \delta_{2}(t)-\beta_{2}^{*}(t) \delta_{1}(t)}{p \beta_{1}^{*}(t)+(1-p) \beta_{2}^{*}(t)} .
$$

The function $\Gamma(p)$ is always monotone in $p \in(0,1)$ and so the monotone convergence theorem can be used to show that

$$
\Delta_{1}=\lim _{c \rightarrow 0} E \log [1+\Gamma(0) \wedge \Gamma(c)]
$$

whenever $\Delta_{1}$ exists as a finite or infinite value. Thus when $\Delta_{1}>0$ there is a positive constant $c$ such that

$$
E \log [1+\Gamma(0) \wedge \Gamma(c)]>0 .
$$

Now if $P_{1}(t) \rightarrow 0$ there is a time $\tau$ such that $P_{1}(t+\tau)<c$ for all $t>0$. However

$$
P_{1}(t+\tau) / P_{1}(\tau) \geqslant \prod_{s=\tau}^{t+\tau-1}\{1+\Gamma(s, 0) \wedge \Gamma(s, c)\} .
$$

From (10) and the strong law of large numbers the RHS $\rightarrow \infty$ as $t \rightarrow \infty$, a.s. 
contradicting the hypothesis that $P_{1}(t+\tau)$ remains below $c$ for all $t$, a.s. Hence $P_{1}(t) \nrightarrow 0$, a.s. Similarly $P_{2}(t) \nrightarrow 0$ a.s. whenever $\Delta_{2}>0$. Thus we have proved the following theorem.

Theorem 3.1. If $\Delta_{i}>0$ then $\mathscr{P}\left(P_{i}(t) \rightarrow 0\right)=0$.

It is possible to have $\Delta_{1}$ and $\Delta_{2}$ simultaneously positive so that with probability 1 neither species density approaches 0 . Under mild additional conditions it is shown in section 5 below that both species persist in the sense of stochastic boundedness, that is they will coexist in the sense accepted in this paper. The interesting feature is that this situation of coexistence is only possible in a variable environment for we have seen above that coexistence does not occur in the lottery model with a constant environment. A study of the kinds of environmental variability leading to coexistence is deferred until section 4 , below, because we must now complete our analysis of the implications of the signs of the boundary growth rates by considering the situations where one or both boundary growth rates are negative.

First, assume that $\Delta_{1}$ is negative. In general

$$
P_{1}(t+1) / P_{1}(t) \leqslant 1+\Gamma(t, 0) \vee \Gamma(t, c)
$$

for $0<P_{1}(t) \leqslant c$. Moreover the monotone convergence theorem shows that

$$
\Delta_{1}=\lim _{c \downarrow 0} E \log \{1+\Gamma(0) \vee \Gamma(c)\}
$$

so that for $c$ sufficiently small the quantity $E \log \{1+\Gamma(0) \vee \Gamma(c)\}$ is a negative upper bound to the mean instantaneous growth rate for $P_{1}(t) \leqslant c$, which is independent of the value of $P_{1}(t)$. The existence of this negative upper bound means that $P_{1}(t) \rightarrow 0$ provided $P_{1}(t)$ is less than $c$ infinitely often. This is stated precisely as the following lemma which is proved in Appendix I as are all lemmas in this section.

Lemma 3.2. If $\Delta_{1}<0$ then there is a $c>0$ such that $E \log \{1+\Gamma(0) \vee \Gamma(c)\}<0$ and for this $c, P_{1}(t) \in(0, c)$ i.o., implies $P_{1}(t) \rightarrow 0$ a.s.

Coming near 0 infinitely often is obviously a necessary condition for convergence to 0 and Lemma 3.2 shows that it is also sufficient when $\Delta_{i}<0$. The following lemma shows that unless $\rho \equiv 1$ the density of at least one of the two species must approach 0 arbitrarily closely, infinitely often, as a result of accumulated fluctuations of the environment.

Lemma 3.3. If $\mathscr{P}(\rho=1)<1$ then, for every $\varepsilon>0$

$$
\mathscr{P}\left(P_{1}(t) \in[\varepsilon, 1-\varepsilon]^{c} \text { i.o. }\right)=1 \text {. }
$$

Unless the $\Delta_{i}$ are both 0 , the hypothesis of Lemma 3.3 will hold. Combining this with Lemma 3.2 it is clear that when $\Delta_{1}<0$ and $\Delta_{2}<0$ either $P_{1}(t) \rightarrow 0$ or $P_{2}(t) \rightarrow 0$, a.s. Moreover it is also clear from the proof of Lemma 3.2 (Appendix I) that $\mathscr{P}\left(P_{i}(t) \rightarrow 0\right)>0, i=1,2$, and so the species density that converges to 0 cannot be predicted with certainty. We shall therefore refer to this situation as random exclusion.

For the remaining case where the $\Delta_{i}$ have opposite signs we need the following lemma which shows that the species with the negative boundary growth rate is the species which experiences arbitrarily low densities infinitely often. 
Lemma 3.4. If $\Delta_{1}>0$ then, for every $\varepsilon>0, \mathscr{P}\left(P_{1}(t) \in(1-\varepsilon, 1)\right.$ i.o. $)=1$.

Combining Lemmas 3.2 and 3.4 we see that $P_{2}(t)$ must converge to 0 when $\Delta_{1}>0$ and $\Delta_{2}<0$. The results we have obtained are summarized as follows.

Theorem 3.5. 1. If $\Delta_{i}>0, i=1,2$ then $\mathscr{P}\left(P_{i}(t) \rightarrow 0\right)=0, i=1,2$.

2. If $\Delta_{i}>0$ and $\Delta_{j}<0$ then $P_{i}(t) \rightarrow 1$, a.s., $P_{j}(t) \rightarrow 0$, a.s.

3. If $\Delta_{i}<0, i=1,2$ then $\mathscr{P}\left(P_{i}(t) \rightarrow 0\right)>0, i=1,2$, and $\mathscr{P}\left(P_{1}(t) \rightarrow 0\right.$ or $\left.P_{2}(t) \rightarrow 0\right)=1$.

This theorem is the first stage in the process of determining the circumstances under which coexistence will occur in the sense that both species are s.b. persistent. In case 3 neither species is s.b. persistent because in this case $\sup _{t} \mathscr{P}\left(P_{i}(t)<p\right)$ $\geqslant \mathscr{P}\left(P_{i}(t) \rightarrow 0\right)>0$ for all positive $p$. In case 2 species $i$ is s.b. persistent while species $j$ is not. In case 1 mild additional conditions are necessary to ensure that both species are s.b. persistent (section 5, below) so that coexistence occurs.

\section{The Effect of Environmental Variability on Boundary Growth Rates and Coexistence}

We assume here that the "mild additional conditions" have been imposed so that coexistence occurs when both boundary growth rates are positive.

For both boundary growth rates to be positive the assumption of overlapping generations $\left(\delta_{i}<1\right)$ is essential for, as we have seen, coexistence does not occur otherwise. The parameter $\rho$ must take on values on either side of 1 , i.e. $\log \rho$ must take both positive and negative values so that each species has periods when it increases or is "favored" by the environment. As might be expected the magnitude of the death rates is an important factor in coexistence for when death rates are large a situation is approached where coexistence is impossible, namely the nonoverlapping generations situation. We shall see, however, that variation in death rates alone is incapable of producing coexistence. Therefore in most of our development we shall take the probability distributions of the death rates as given and see how coexistence is affected by different levels of variation in $\log \rho$, resulting from different levels of variation in the birth-competition parameters, the $\beta_{i}^{*}$. We also assume that $E \log \left(1-\delta_{i}\right)>-\infty$ so that death rates do not approach 1 too frequently.

For fixed death rate distributions, coexistence always results from sufficient variation of $\log \rho$ about 0 . Indeed coexistence will occur provided only that the favorable periods are sufficiently favorable, as measured by the magnitude of $\log \rho$, regardless of how unfavorable the unfavorable periods might be. This remarkable result can be seen from the following inequalities which are derived in Appendix 2.

$$
\begin{aligned}
& \Delta_{1} \geqslant E \delta_{1}(\log \rho)^{+}+E \log \left(1-\delta_{1}\right) 1_{\{\rho<1\}} \\
& \Delta_{2} \geqslant E \delta_{2}(\log \rho)^{-}+E \log \left(1-\delta_{2}\right) 1_{\{\rho>1\}} .
\end{aligned}
$$

Note that $(\log \rho)^{+}=0 \vee \log \rho$ and $(\log \rho)^{-}=0 \vee(-\log \rho)$. The quantity $(\log \rho)^{+}$ is 0 during unfavorable periods for species $l$ and if it is sufficiently large during favorable periods, species 1 will persist. Similarly species 2 persists if $(\log \rho)^{-}$is 
sufficiently large during species 2's favorable periods. In the case of small constant death rates these statements can be made quite precise for then (14) implies that $\Delta_{1}$ and $\Delta_{2}$ will be positive respectively when the following inequalities hold

$$
E\left[(\log \rho)^{+} \mid \rho>1\right]>\mathscr{P}(\rho<1) / \mathscr{P}(\rho>1)
$$

and

$$
E\left[(\log \rho)^{-} \mid \rho<1\right]>\mathscr{P}(\rho>1) / \mathscr{P}(\rho<1) .
$$

Thus species 1 (species 2$)$ persists if the mean of $(\log \rho)^{+}\left((\log \rho)^{-}\right)$during favorable periods is greater than the odds of an unfavorable period. These sufficient conditions for coexistence can be satisfied in the presence of arbitrarily large mean differences in the extents to which the environment favors each of the two species as measured by the natural measure $\mu=E \log \rho=E(\log \rho)^{+}-E(\log \rho)^{-}$. A natural measure of variation in $\log \rho$ about 0 is given by $E(\log \rho)^{+} \wedge E(\log \rho)^{-}$. If this quantity is greater than 1 then the inequalities (15) will be satisfied and coexistence will occur. In the general case, the boundary growth rates will be positive if $E(\log \rho)^{+} \wedge E(\log \rho)^{-}>E \log \delta_{i}\left(1-\delta_{i}\right), i=1,2$ (Appendix 2, inequality (A3)). This latter condition is highly conservative but it still indicates the fundamental role of $E(\log \rho)^{+} \wedge E(\log \rho)^{-}$.

We have found sufficient but by no means necessary conditions for coexistence. To obtain conditions which are both necessary and sufficient the boundary growth rates must be calculated more precisely. This is done numerically in Chesson and Warner (1981). Alternatively one can use the approximate formulae given there and in Appendix 2 for the case of small death rates. However qualitative insights into the roles of various factors affecting coexistence do not require such calculations and we now turn our attention to such insights. In seeing how various factors affect coexistence we shall generally regard an increase in some factor as favorable if it increases both boundary growth rates.

The tendency of the $\Delta_{i}$ to increase with variation in $\log \rho$ comes about largely from the convexity of $\log \left[1+\delta_{1}(\rho-1)\right]$ as a function of $\log \rho$. The case of constant death rates, but variable $\log \rho$, is especially illuminating for then Jensen's inequality implies that

$$
\Delta_{1}=E \log \left\{1+\delta_{1}(\rho-1)\right\}>\log \left\{1+\delta_{1}\left(e^{\mu}-1\right)\right\}
$$

and

$$
\Delta_{2}=E \log \left\{1+\delta_{2}\left(\rho^{-1}-1\right)\right\}>\log \left\{1+\delta_{2}\left(e^{-\mu}-1\right)\right\} .
$$

Thus variation in $\log \rho$ increases both boundary growth rates over the values that would obtain in a constant environment with $\log \rho \equiv \mu$, the mean advantage. Moreover it follows from our results below that the differences between the LHSs and RHSs of (16) increase with variation in $\log \rho$. For $\mu=0$ the RHSs of (16) are 0 so that any variation at all in $\log \rho$ is sufficient for both $\Delta_{i}$ to be positive.

To see how the $\Delta_{i}$ increase with increasing variability we return to the general case where the $\delta_{i}$ are possibly variable and we suppose that $\log \rho$ is naturally divisible into additive components of variation. We write $\log \rho=\log R+X$ where $X$ is the component of variation to be increased. For example $X$ could be $\log \beta_{1}^{*} / \beta_{2}^{*}-E \log \beta_{1}^{*} / \beta_{2}^{*}$ representing variation in birth-competition. To justify 
various limits below we assume $E|\log \rho|<\infty$ and $E|X|<\infty$, but we do not assume that $X$ has mean 0 , only that $X$ takes both positive and negative values. To increase variability in $X$ we write $X=\sigma X^{\prime}$ where $X^{\prime}$ is some given random variable not depending on $\sigma$ and for definiteness we assume that $E\left(X^{\prime}\right)^{+} \wedge E\left(X^{\prime}\right)^{-}=1$ so that $\sigma=E X^{+} \wedge E X^{-}$, the natural measure of variation about 0 introduced above.

The $\Delta_{i}$ are averages of strictly convex functions of $\sigma$ and therefore the $\Delta_{i}$ are themselves strictly convex functions of $\sigma$. Moreover

$$
\frac{d \Delta_{1}}{d \sigma}=E \frac{\delta_{1} R X^{\prime} e^{\sigma X^{\prime}}}{1+\delta_{1}\left(R e^{\sigma X^{\prime}}-1\right)} .
$$

As a consequence of convexity the $\left(d \Delta_{i} / d \sigma\right)$ are increasing functions of $\sigma$. To see that they are eventually positive we note that

$$
\frac{d \Delta_{1}}{d \sigma} \rightarrow E\left(X^{\prime}\right)^{+} \quad \text { and } \quad \frac{d \Delta_{2}}{d \sigma} \rightarrow E\left(X^{\prime}\right)^{-}
$$

giving the surprising result that $X$ is ultimately the only factor involved in the rates of increase of the $\Delta_{i}$. An even more remarkable consequence of this is that $\Delta_{1} \sim E X^{+}$and $\Delta_{2} \sim E X^{-}$for large $\sigma$ so that $X$ emerges as the dominant factor in the $\Delta_{i}$ as the variability specified by $X$ increases. Since we are free to choose $X=\log \rho$, this shows that $\Delta_{1} \sim E(\log \rho)^{+}$and $\Delta_{2} \sim E(\log \rho)^{-}$. These asymptotic expressions are generally quite poor approximations to the actual values but they do indicate interesting trends. For example these expressions show that the $A_{i}$ tend to lose any dependence on the $\delta_{i}$ that is not already accounted for in $\log \rho$. The lower bounds (14) seem to indicate that the $\Delta_{i}$ are approximately proportional to the $\delta_{i}$ when $\log \rho$ is highly variable, but this is clearly not correct.

The results above show that the $\Delta_{i}$ are strictly increasing in $\sigma$ for sufficiently large $\sigma$. For variation this large, any further increase, whether small or large will favor coexistence. However the $\Delta_{i}$ may decrease before they increase so that for some components of variability, some increases in $\sigma$ will not favor coexistence and could lead to competitive exclusion. However there is a natural class of situations where any increase in $\sigma$ will increase the $\boldsymbol{A}_{i}$. This occurs when we choose to increase variation orthogonal to some particular factor or set of factors so that the effect of these factors is kept constant. Let $Y$ be one of these factors and define $\log R=E\left[\log \rho \mid Y, \delta_{1}, \delta_{2}\right]$ and $X=\log \rho-E\left[\log \rho \mid Y, \delta_{1}, \delta_{2}\right]$. Increasing variation in $X$ therefore increases variation orthogonal to $Y, \delta_{1}, \delta_{2}$. Since $E\left[X^{\prime} \mid R, \delta_{1}, \delta_{2}\right]=0$ we have $\left.\left(d \Delta_{i} / d \sigma\right)\right|_{\sigma=0}=0$. The strict convexity of the $\Delta_{i}$ now implies that the $\Delta_{i}$ are also strictly increasing functions of $\sigma$. Thus small, as well as large, increases in the variation in $X$ are always favorable to coexistence.

Example 1. Let $Y \equiv 0$, then $X$ is variation orthogonal to death rate variation. Thus it is variation in $\log \rho$ due to birth-competition with the effect of covariation with death rates removed. $E\left[\log \beta_{1}^{*} / \beta_{2}^{*} \mid \delta_{1}, \delta_{2}\right]$ is unaffected by $\sigma$ and the variation in $\log \beta_{1}^{*} / \beta_{2}^{*}$ is increased about this conditional mean value.

Example 2. Let $Y=\beta_{1}^{*}$. In this case the $\Delta_{i}$ increase as variation in $\log \beta_{2}^{*}$ is increased about $E\left[\log \beta_{2}^{*} \mid \beta_{1}^{*}, \delta_{1}, \delta_{2}\right]$.

Example 3. Let $Y$ be some environmental factor like temperature known to affect 
the $\beta_{i}^{*}$ in a certain way. Increasing variation in $X$ then corresponds to increasing variation in $\log \rho$ with the effect of temperature variation kept constant.

In these examples the $\Delta_{i}$ are strictly increasing in $\sigma$. It follows that a higher value of $\sigma$ allows coexistence for a wider range of possibilities for $X^{\prime}, R, \delta_{1}$ and $\delta_{2}$. For example, for a given $\sigma$ let $A_{\sigma}$ be the set of possible probability distributions of $\left(X^{\prime}, R, \delta_{1}, \delta_{2}\right)$ having the property that the $\Delta_{i}$ are both positive. Then $A_{\sigma}$ is a monotonically increasing family. This suggests that coexistence is possible under broader circumstances with larger $\sigma$.

The method above deals only with components of variation orthogonal to death rates. In other cases it is necessary to consider the nature of the statistical dependence between the death rates and the component of variation. For example suppose

$$
X=\log \beta_{1}^{*} / \beta_{2}^{*}-E \log \beta_{1}^{*} / \beta_{2}^{*}
$$

and let

$$
\lambda=\exp \left\{E \log \beta_{1}^{*} / \beta_{2}^{*}\right\}
$$

then

$$
\left.\frac{d \Delta_{1}}{d \sigma}\right|_{\sigma=0}=E \frac{\lambda \tau_{1} X^{\prime}}{1+\lambda \tau_{1}}
$$

where $\tau_{i}=\delta_{j} /\left(1-\delta_{i}\right)$ for $i \neq j$. Any increase in $\sigma$ will favor coexistence if $\left.\left(d \Delta_{i} / d \sigma\right)\right|_{\sigma=0}$ is nonnegative for $i=1,2$. This will be so if $X^{\prime}$ is correlated nonnegatively with $\tau_{1} /\left(1+\lambda \tau_{1}\right)$ and nonpositively with $\tau_{2} /\left(1+\lambda^{-1} \tau_{2}\right)$, i.e. $X^{\prime}$ must tend to increase with $\tau_{1}$ and decrease with $\tau_{2}$. These conditions become a little more interpretable for small death rates for then

$$
\left.\frac{d \Delta_{1}}{d \sigma}\right|_{\sigma=0} \approx E \lambda \delta_{2} X^{\prime}
$$

and coexistence is favored by any increase in $\sigma$ when $X^{\prime}$ is positively correlated with $\delta_{2}$ and negatively correlated with $\delta_{1}$. This requires a tendency for a species to experience relatively favorable periods for birth-competition while the other species experiences high death rates. When the reverse conditions hold small increases in $\sigma$ will be unfavorable to coexistence. However in all cases, sufficiently large increases in $\sigma$ continue to favor coexistence.

Correlations between birth-competition and death rates may also permit coexistence to be favored by increased variation in death rates. For example Chesson and Warner (1981) show that the boundary growth rates will increase as functions of the variances of the death rates under the assumptions that death rates are small and $\delta_{j}$ is positively correlated with $\beta_{i}^{*} / \beta_{j}^{*}$ for $i \neq j$. This latter condition differs slightly from the condition above for the $\Delta_{i}$ to increase as functions of increasing variability in $\log \beta_{i}^{*} / \beta_{j}^{*}$.

It does not appear that death rate variation can promote coexistence in the absence of correlations with birth-competition for when birth-competition does not vary, Jensen's inequality implies that

$$
\Delta_{1} \leqslant \log \left\{1-E \delta_{1}+\left(E \delta_{2}\right) \beta_{1}^{*} / \beta_{2}^{*}\right\}
$$


and

$$
\Delta_{2} \leqslant \log \left\{1-E \delta_{2}+\left(E \delta_{1}\right) \beta_{2}^{*} / \beta_{1}^{*}\right\} .
$$

These upper bounds on the $\Delta_{i}$ cannot both be positive so that coexistence cannot result from death rate variation alone. Indeed large variation in death rates can be quite unfavorable to coexistence, for example Chesson and Warner (1981) study the effect of increasing variation in $\left(\delta_{1}, \delta_{2}\right)$ so that values near $(0,1)$ and $(1,0)$ are assumed with some fixed probability. The variation in birth-competition is kept constant and it is found that both boundary growth rates eventually take on negative values. Thus a situation of random exclusion of one of the two species occurs.

That large variations in death rates may lead to competitive exclusion is not surprising when it is realized that large death rates permit large declines in population density during unfavorable periods. Indeed, coexistence is generally more likely when death rates are smaller, for Chesson and Warner (1981) have shown that negative boundary growth rates may become positive when death rates are uniformly decreased, while positive growth rates must remain positive.

\section{Summary of Conditions for Coexistence}

We have found that overlapping generations and variation in birth-competition are essential requirements for coexistence. Without overlapping generations, coexistence is impossible and as the degree of overlap in generations is increased, i.e., as death rates are decreased, coexistence becomes more likely.

Coexistence requires that $\log \rho$ must vary about 0 and this variation must result at least in part from variation in birth-competition. Variability in $\log \rho$ about 0 is measured by $E(\log \rho)^{+} \wedge E(\log \rho)^{-}$. For given death rate distributions, satisfying mild assumptions, it is always possible to find a minimum level of variability about 0 , for $\log \rho$, such that coexistence occurs at all higher levels of variability. This minimum level of variability depends only on the death rate distributions and so is independent of both the mean advantage that one species has over another and the shape of the distribution of $\log \rho$.

We have identified a class of methods of increasing variability in $\log \rho$ such that both boundary growth rates increase monotonically and unboundedly with increasing variability. In these circumstances any increase in variability is favorable to coexistence because both species will tend to decline more slowly, or increase more quickly, at low density. Moreover, a sufficiently large increase in variability will result in coexistence and no increase in variability can change coexistence into competitive exclusion. In these circumstances it follows that higher levels of variability permit coexistence in a broader range of situations.

Such monotonic increases in boundary growth rates occur whenever the component of variation is chosen to be that part of $\log \rho$ orthogonal to a given set of factors, which include the death rates. Monotonic increases will still occur for other additive components of variation in $\log \rho$ provided they are appropriately correlated with death rates and the remainder of $\log \rho$. Moreover, increasing any additive component of variation in $\log \rho$ will ultimately result in monotonic and unbounded increases in the $\Delta_{i}$ so that coexistence is the eventual result. This occurs 
whether or not the component of variation has mean 0 so that the mean advantage can increase at the same rate as variation about the mean advantage and still lead to coexistence. The reason for this is that $E(\log \rho)^{+} \wedge E(\log \rho)^{-}$can increase when $|E \log \rho|$ and $|\log \rho-E \log \rho|$ are increased at the same rate. However if $|E \log \rho|$ is increased at a faster rate than $|\log \rho-E \log \rho|$ then $E(\log \rho)^{+} \wedge E(\log \rho)^{-}$will decrease and exclusion will be the final outcome.

In all of the above, death rate distributions are held constant and variability is increased through birth-competition. The effect of increasing death rate variability is complex. When death rates are small, increased variability in death rates will be favorable or unfavorable to coexistence depending on the correlations between death rates and birth-competition. Without variation in birth-competition, death rate variability cannot lead to coexistence. However, increasing death rate variability may lead to exclusion regardless of the presence of birth-competition variability. For example, random exclusion results from increasing variation in $\left(\delta_{1}, \delta_{2}\right)$ so that values near $(0,1)$ and $(1,0)$ are assumed.

Our investigation of conditions for coexistence is best regarded as an exploration of the set of probability distributions of $\left(\beta_{1}^{*}, \delta_{1}, \beta_{2}^{*}, \delta_{2}\right)$ for which both boundary growth rates are positive. This set, which we shall call the coexistence set, is very complex and is not usefully described by a few parameters. Thus the set cannot be mapped precisely in any useful way and we must be content with special sections through the set (e.g., Figure 1 in Chesson and Warner 1981) or we can use the sort of results obtained here to do the following.

1. Identify large subsets of the coexistence set and large subsets of its complement.

2. Find a set of rules showing how to modify a given distribution so that

(a) the coexistence set is entered;

(b) another distribution in the coexistence set is obtained;

(c) the coexistence set is left;

(d) the coexistence set is not entered.

Although not written formally as a set of rules, our investigations do provide such rules, thus they establish lines of comparison. For example, with relatively invariant death rate distributions, a gradient of increased variation of $\log \rho$ about 0 should lead towards coexistence. Whether or not this trend is monotonic depends both on the amount of variability at the low end of the gradient and the correlations between birth-competition and death rates.

\section{A More Specific Question}

The question, "What are the general features of the coexistence set?" led to the methods of increasing variability discussed above. However, sometimes the focus is on a specific method of increasing variability with the question then being "how does it intersect the coexistence set?" For example, a natural question is, "How does the situation of a constant reproductive output per individual, per unit time, compare with the situation of a varying reproductive output with the same mean?" The important feature is that the mean reproductive output is specified as the same in the two situations. The two situations will thus involve the same total 
reproductive effort but with a different distribution over time. To investigate this question we need to keep $E \beta_{i}^{*}$ constant but alter the variation in $\beta_{i}^{*}$ about $E \beta_{i}^{*}$. Even with this relatively narrow question there are still many ways of increasing variation. The following method is chosen on the basis of tractability.

Define

$$
\log \beta_{i}^{*}=\log \bar{\beta}_{i}^{*}+\sigma X_{i}^{\prime}-\phi_{i}(\sigma)
$$

where $\bar{\beta}_{i}^{*}=E \beta_{i}^{*}, E X_{i}^{\prime}=0$ and $\phi_{i}(\sigma)=\log E e^{\sigma X_{i}^{\prime}}$. As $\sigma$ is increased the variance of $\beta_{i}^{*}$ increases but the mean remains constant. This increase in variance is accompanied by a change in shape of the distribution. However a change in shape, with an increase in variance about a fixed mean, is not unreasonable because normally one would expect the distribution of $\beta_{i}^{*}$ to have positive density over the entire range $(0, \infty)$. Under these circumstances a change in shape must accompany the increase in variance.

To analyze the model we first consider the situation where death rates are small and non-random so that the following approximation (Chesson and Warner, 1981) is applicable:

$$
\Delta_{i}=\delta_{2} E\left(\beta_{1}^{*} / \beta_{2}^{*}\right)-\delta_{1}+o\left(\delta_{1}+\delta_{2}\right)
$$

Now

$$
E\left(\beta_{1}^{*} / \beta_{2}^{*}\right)=\left(\bar{\beta}_{1}^{*} / \bar{\beta}_{2}^{*}\right) e^{\psi(\sigma)+\phi_{2}(\sigma)-\phi_{1}(\sigma)}
$$

where $\psi(\sigma)=\log E e^{\sigma\left(X_{1}^{\prime}-X_{2}^{\prime}\right)}$. Thus the boundary growth rates are both increasing functions of $\sigma$ if and only if $\psi(\sigma)+\phi_{2}(\sigma)-\phi_{1}(\sigma)$ and $\psi(-\sigma)+\phi_{1}(\sigma)-\phi_{2}(\sigma)$ are both increasing functions of $\sigma$. This condition is not greatly restrictive and essentially requires the variation in $X_{1}^{\prime}-X_{2}^{\prime}$ to exceed the difference in the variation of $X_{1}^{\prime}$ and $X_{2}^{\prime}$ separately. Rather than attempt to make this statement more precise we shall just give the following illustrations.

Let $\left(X_{1}^{\prime}, X_{2}^{\prime}\right)$ be bivariate normal, then $\phi_{i}(\sigma)=\frac{1}{2} \sigma^{2} \mathscr{V} X_{i}^{\prime}$ and $\psi(\sigma)=$ $\frac{1}{2} \sigma^{2} \mathscr{V}\left(X_{1}^{\prime}-X_{2}^{\prime}\right)$. The $\Delta_{i}$ are both unbounded increasing functions of $\sigma$ provided

$$
\mathscr{V}\left(X_{1}^{\prime}-X_{2}^{\prime}\right)>\left|\mathscr{V} X_{1}^{\prime}-\mathscr{V} X_{2}^{\prime}\right|
$$

so that the "variation" spoken of above is given by the variance in this example. Inequality (25) is always satisfied when $X_{1}^{\prime}$ and $X_{2}^{\prime}$ are nonpositively correlated. When $X_{1}^{\prime}$ and $X_{2}^{\prime}$ have positive correlation, $\gamma$ say, then this correlation places the restriction $\gamma^{2}<\mathscr{V} X_{1}^{\prime} / \mathscr{V} X_{2}^{\prime}<\gamma^{-2}$ on how much $\mathscr{V} X_{1}^{\prime}$ and $\mathscr{V} X_{2}^{\prime}$ may differ if (25) is to hold and coexistence is to be favored by increasing variation.

The significant features of this example are that there are broad situations where increases in $\sigma$ do favor coexistence and also broad situations where they do not. However, we should note that the approximation (23) breaks down as $\sigma$ is increased beyond some level depending on the magnitude of the death rates. The approximation does hold uniformly for finite intervals of $\sigma$ and so the conclusions above apply for $\sigma$ in some finite range. This range increases to $\infty$ as the death rates decrease to 0.

To see what happens when death rates and $\sigma$ have no restriction, note that the mean advantage $\mu=$ constant $+\phi_{2}(\sigma)-\phi_{1}(\sigma)$. The $\phi_{i}$ are convex functions of $\sigma$ 
and only in the case where $X_{i}^{\prime}$ has a finite upper bound $m_{i}$ does $\phi_{i}(\sigma) / \sigma$ converge to a finite limit. This leaves open the possibility that $\mu / \sigma$ is unbounded as $\sigma$ increases and indeed this is so when $X_{1}^{\prime}$ and $X_{2}^{\prime}$ are normal with different variances. If $|\mu / \sigma| \rightarrow \infty$ we must have $E(\log \rho)^{-} \wedge E(\log \rho)^{+} \rightarrow 0$ since $|\log \rho-\mu| / \sigma$ does not increase with $\sigma$ so that there must be a decline in the variation of $\log \rho$ about 0 . Thus, in the normal case with unequal variances, exclusion is the eventual result of increasing the variances of the birth-competition parameters while their means are kept constant. This is clearly also true in a great variety of other cases.

Combining the results above with those for small death rates, it can be seen that there are broad situations where increasing the variances of the birth-competition parameters initially leads to increasing boundary growth rates and possibly coexistence but eventually one of the boundary growth rates decreases leading to competitive exclusion.

There are at least two easily identified cases where coexistence is the ultimate result of increasing the variances of the $\beta_{i}^{*}$ 's while their means remain constant. If the $X_{i}^{\prime}$ have finite upper bounds $m_{i}$ then $(\log \rho) / \sigma \rightarrow\left(X_{1}^{\prime}-X_{2}^{\prime}\right)-\left(m_{1}-m_{2}\right)$ so provided this quantity takes both positive and negative values, the boundary growth rates increase to $+\infty$. Also when $X_{1}^{\prime}$ and $X_{2}^{\prime}$ are identically distributed, $\mu$ does not change with $\sigma$ and our work on additive components of $\log \rho$ applies to show that the $\Delta_{i}$ again increase to $+\infty$. These examples are quite particular but they are given in the expectation that they reflect approximately the results for the many useful cases near them.

We have a mixed set of results for increasing variances of the $\beta_{i}^{*}$ while the means remain constant. Increasing variances may initially be unfavorable to coexistence, or it may be favorable initially but unfavorable ultimately, or favorable both initially and ultimately, depending on the precise circumstances. However one thing is clear: coexistence can only occur if at least one of the $\beta_{i}^{*}$ has nonzero variance. Thus if variances are increased from zero to positive values, there is no doubt that the breadth of situations permitting coexistence is increased. Can a statement like this be made for general increases in the variances of the $\beta_{i}^{*}$ ? This is an important question for when comparing two field situations we are always dealing with changes in more than one factor. A conclusion that coexistence will occur if variances are increased, while everything else is being held constant, is perhaps less useful than the conclusion that there are a greater variety of conditions under which coexistence can occur with higher variances.

To see if a greater breadth of values of the means $\bar{\beta}_{i}^{*}$ is consistent with coexistence when variances are higher, we recall that the sets $A_{\sigma}$, in our discussion of additive components of $\log \rho$, are monotone increasing. It follows that the intervals $\left(a_{\sigma}, b_{\sigma}\right)$ of values of the mean advantage $\mu$ which permit coexistence for a given value of $\sigma$, are an increasing set of intervals. Expressing this in terms of values of $\log \bar{\beta}_{1}^{*} / \bar{\beta}_{2}^{*}$ we see that coexistence occurs when $\log \bar{\beta}_{1}^{*} / \bar{\beta}_{2}^{*} \in\left(a_{\sigma}+f(\sigma), b_{\sigma}+f(\sigma)\right)$ with $f(\sigma)=\phi_{1}(\sigma)-\phi_{2}(\sigma)+E \log \delta_{1} / \delta_{2}$. The length of the interval is an increasing function of $\sigma$ and for this reason one could conclude that a broader range of $\left(\bar{\beta}_{1}^{*} / \bar{\beta}_{2}^{*}\right)$ values is permitted with larger variances. However since the intervals are not necessarily increasing in the set theoretic sense, this conclusion is arguable. A different measure of breadth of the permissible $\left(\bar{\beta}_{1}^{*}, \bar{\beta}_{2}^{*}\right)$ values could lead to a different conclusion. We are thus led to the difficult problem of deciding the 
biologically most appropriate measure of breadth, which we shall not attempt to do here.

\section{Stochastic Boundedness with Positive Boundary Growth Rates}

In our study above of the manner in which environmental variability may lead to positive boundary growth rates we have assumed that such positive rates are essentially equivalent to coexistence. If both boundary growth rates are positive both species tend to increase from low densities and both have zero probability of converging to 0 . Are both species also s.b. persistent? We know that convergence to 0 with probability 0 is not sufficient for s.b. persistence because the nonoverlapping generations model with 0 mean advantage does not give s.b. persistence yet does give 0 probability of convergence to 0 . However, the nonoverlapping generations model with 0 mean advantage does not satisfy the invasibility criterion, i.e., does not have positive boundary growth rates. Is invasibility sufficient for s.b. persistence? The answer here is also no for as we shall see s.b. persistence depends on how violently a population is returned to low densities as well as its power of recovery at low densities. To demonstrate s.b. persistence when both boundary growth rates are positive we need to impose mild additional conditions which ensure that population declines are not too violent. First we demonstrate by counter example that invasibility does not imply s.b. persistence.

Let $N(t)$ be population density and assume the following model of geometric growth followed by randomly determined crashes from high density (a crude model of some small mammal populations):

$$
\begin{array}{lll}
N(t+1)=2 N(t) & \text { for } & N(t)<K \\
N(t+1)=U(t) N(t) & \text { for } & N(t) \geqslant K .
\end{array}
$$

The random variable $U(t)$ takes values in the internal $(0,1)$ and $U(0), U(1), \ldots$, will be assumed i.i.d. Regardless of the distribution of $U(t), N(t)$ increases at low values and so the invasibility criterion is satisfied. Moreover $\mathscr{P}(N(t) \rightarrow 0)=0$. However in some situations $N(t)$ converges in probability to 0 and so the population certainly does not persist in the sense of stochastic boundedness. To provide the simplest example of this assume that $\log _{2} N(0)$ and $\log _{2} U(t)$ are both integers. Then $\log _{2} N(t)$ is a homogeneous Markov chain with integer state space. If $E \log _{2} U(t)=-\infty$ (a model of occasional very severe crashes) then $\log _{2} N(t)$ is null recurrent because all states are recurrent but the expected return times are infinite. This null recurrence implies that $\mathscr{P}\left(\log _{2} N(t)>x\right) \rightarrow 0$ as $t \rightarrow \infty$, for all $x$, and it follows that $N(t)$ converges in probability to 0 . Thus the population is not s.b. persistent.

To prove stochastic boundedness for the lottery model we must preclude the kind of violent crashes seen in the above example. This is done by assuming that there is a positive number $\varepsilon$ such that

$$
E\left(1-\delta_{i}\right)^{-\varepsilon}<\infty
$$

$i=1,2$. To translate this into a condition on population fluctuations we note that

$$
P_{i}(t+1) / P_{i}(t) \geqslant 1-\delta_{i}(t)
$$


and so (26) implies

$$
E\left[\left\{P_{i}(t+1) / P_{i}(t)\right\}^{-\varepsilon} \mid P_{i}(t)\right] \leqslant K<\infty
$$

where $K=E\left(1-\delta_{1}\right)^{-\varepsilon} \vee E\left(1-\delta_{2}\right)^{-\varepsilon}$, and we assume in (27), as we do below, that $\mathscr{E}$ is an i.i.d. process.

Theorem 5.1. Under the above assumptions both species 1 and species 2 are s.b. persistent whenever $\Delta_{1}$ and $\Delta_{2}$ are both positive.

The proof is given in Appendix 3 and uses the conditions above to prove that $E P_{i}^{-\varepsilon}(t)$ remains bounded as $t \rightarrow \infty$, for some positive $\varepsilon$. Stochastic boundedness follows from this.

We have seen that the situation $\Delta_{1}>0, \Delta_{2}>0$ is favored by certain kinds of environmental variability. Also, in this situation, both species will increase when rare and both species densities have 0 probability of converging to 0 . However as shown above these latter properties are consistent with drift of the probability distribution of $P_{i}(t)$ towards 0 densities, when severe stochastic population fluctuations are present. Theorem 5.1 shows that such drift of probability to 0 cannot occur here when condition (26) is imposed. Consequently the sort of coexistence obtained here from a stochastic mechanism is as strong as one would expect in a situation where coexistence is explained by a deterministic mechanism operating in a stochastic environment (e.g. Turelli and Gillespie, 1980). This conclusion is further strengthened below. First it is shown that in addition to s.b. persistence, the population densities converge in distribution to positive random variables and then in the next section the properties of this distribution are studied for an interesting limiting case.

Theorem 5.2. Under the hypothesis of Theorem 5.1, the distribution of $P_{i}(t)$ converges as $t \rightarrow \infty$ to a distribution on $(0,1)$ which is independent of the distribution of $P_{i}(0)$.

The proof given in Appendix 3 follows Norman (1975) using the fact that $P_{i}(t+1)$ is nondecreasing as a function of $P_{i}(t)$ for given environmental parameters.

Convergence in distribution to a positive random variable is a commonly used persistence criterion (e.g., May, 1974). The limiting distribution obtained in Theorem 5.2 is the unique stationary distribution of the Markov chain $\left\{P_{i}(t), t=0,1, \ldots\right\}$. If the system has been in existence long enough, $P_{i}(t)$ will have this stationary distribution and the quantiles of the limiting distribution will be equal to the stochastic bounds $\xi_{p}$ and can be used for a quantitative assessment of the strength of the s.b. persistence in the same way that the rate of return to equilibrium, and the size of the domain of attraction of an equilibrium, provide quantitative assessments of the strength of stability in a deterministic framework. The variability in population density, expressed by the stationary distribution, gives the magnitude of population fluctuations and indicates how frequently low population densities occur. In the present situation, where the density variables are equivalent to relative frequencies, a bell-shaped stationary distribution will describe a much more stable situation than a $U$-shaped stationary distribution because low and high densities will be seen much less frequently. A detailed discussion of these ideas is given below in the section on stochastic boundedness and finite populations. 
Finding the stationary distribution is a difficult problem in general, but its properties may be determined in certain useful limiting situations and this is the subject of the next section.

\section{Population Fluctuations in the Presence of Extreme Environmental Variability}

Coexistence requires $\log \rho$ to vary about 0 , and this variation must result in part from variation in the ratio of the birth-competition parameters $\beta_{1}^{*} / \beta_{2}^{*}$. In the limiting case of infinite variability, this ratio takes only the values 0 and $\infty$ and then a substantial amount of information can be found concerning the stationary distribution of the process. As variability approaches infinity the model assumes the limiting form

$$
P_{1}(t+1)=\left[1-\delta_{1}(t)\right] P_{1}(t)+\left[\delta_{1}(t) P_{1}(t)+\delta_{2}(t) P_{2}(t)\right] Y(t)
$$

where $I(t)$ takes only the values 0 and 1 and is independent of $P_{i}(t)$. According to (28), $P_{i}(t)$ remains in $(0,1)$. Moreover, under the assumption that $\mathscr{E}$ is an i.i.d. process, Theorems 5.1 and 5.2 apply to show that $\left\{P_{i}(t)\right\}$ has a stationary distribution on $(0,1)$. Thus we see that infinite variability in birth rates does not lead to infinite variability in adult population density, indeed the adult population density fluctuates in a stable manner. We now take up the study of the stationary distribution for this case of infinite variability with the hope that its behavior generally reflects the situation where variability is large (so that the stationary distribution exists) but less than infinite.

To simplify notation we note that (28) is of the general form

$$
P_{1}(t+1)=a(t)+b(t) P_{1}(t)
$$

where $(a(t), b(t)), t=0,1, \ldots$ is an i.i.d. process. It follows that

$$
E P_{1}(t+1)=E a+E b E P_{1}(t)
$$

and

$$
\mathscr{W} P_{1}(t+1)=E b^{2} \mathscr{V} P_{1}(t)+\mathscr{V} a+2 E P_{1}(t) \mathscr{C}(a, b)+\left(E P_{1}(t)\right)^{2} \mathscr{V} b
$$

Defining $\lambda$ and $\nu^{2}$ to be the mean and variance of the stationary distribution, we obtain

$$
\lambda=\frac{E a}{1-E b}=\frac{E \delta_{2} I}{E \delta_{1}(1-I)+E \delta_{2} I}
$$

and

$$
v^{2}=\frac{\mathscr{V}\left[\delta_{2} I(1-\lambda)-\delta_{1}(1-I) \lambda\right]}{2 E\left[\delta_{1}(1-I)+\delta_{2} I\right]-E\left[\delta_{1}(1-I)+\delta_{2} I\right]^{2}} .
$$

In the special case where $\delta_{1}=\delta_{2}=\delta$, and $\delta$ is nonrandom, these formulae reduce to

$$
\lambda=\mathscr{P}(I=1)
$$

and

$$
v^{2}=\frac{\delta}{2-\delta} \hat{\lambda}(1-\lambda)
$$


In (34) we see that the variance decreases with the adult death rate $\delta$ while the mean (33) is unaffected. Even though birth rates are subject to infinite variability we see that variability in the adult population is strictly constrained by the death rate and in fact can be made arbitrarily small by decreasing the death rate. This conclusion can be seen to apply generally to this model for infinite variability by defining $\delta_{i}=\theta \delta_{i}$ where $\theta$ is a nonrandom parameter permitting scaling of the death rates. Expression (31) for $\lambda$ is independent of $\theta$ while expression (32) for $v^{2}$ is of order $\theta$. Thus small death rates lead to small variance. This result provides a quantitative supplement to our previous finding that coexistence can only occur when generations are overlapping. We now find that the greater the overlap in generations, the more stable the coexistence because mean square fluctuations about the mean are less. The death rates $\delta_{i}$ do not figure prominently in the boundary growth rates with extreme variability for we have found $\Delta_{1} \sim E(\log \rho)^{+}$ and $\Delta_{2} \sim E(\log \rho)^{-}$but the results here show that they remain a very important factor in population fluctuations.

More detailed information about population fluctuations can be found for the case where the $\delta_{i}$ are equal and nonrandom for then the shape of the stationary distribution can also be determined and this gives very useful information about the behavior of the stochastic bounds near 0 and 1 . For this special case the stationary distribution is that of the random variable

$$
P=\sum_{i=0}^{\infty} \delta(1-\delta)^{t} I(t) .
$$

When $\delta=\frac{1}{2}$ and $\lambda=\frac{1}{2}$ (i.e. $\mathscr{P}(I(t)=1)=\frac{1}{2}$ ) it is well known that $P$ is uniformly distributed on $(0,1)$. In other cases the distribution is not so simple, indeed the distribution may be singular with respect to Lebesgue measure. For example, the distribution will be singular for $\lambda \neq \frac{1}{2}$ and $\delta=\frac{1}{2}$, or whenever $1-\delta=1 / \mathrm{s}$ for integers $s>2$; and so we cannot always describe the shape of the distribution in terms of its probability density for it may not have one. Thus we introduce some shape terminology applicable to the distribution function $F(p)=\mathscr{P}(P \leqslant p)$. This terminology is a direct extension of the terminology for distributions with densities.

The distribution will be said to have an infinite mode at 0 if $(1 / p) F(p) \rightarrow \infty$ as $p \rightarrow 0$, and an infinite mode at 1 if $1 /(1-p) \cdot[1-F(p)] \rightarrow \infty$ as $p \rightarrow 1$. When such modes occur, one or both species will be found frequently in low numbers and so such situations are not ones of high stability. If a probability density exists but approaches 0 as $p \rightarrow 0(p \rightarrow 1)$ we have $F(p)=o(p)$ as $p \rightarrow 0(1-F(p)=o(1-p)$ as $p \rightarrow 1$ ). We say that the distribution is thin at 0 (at 1$)$. When the distribution is thin at both 0 and 1 the species coexistence is a relatively stable one because small population sizes will occur very infrequently.

To determine these properties for the distribution of $P$ we note that $P$ is always continuous even though not always absolutely continuous. Thus $\mathscr{P}(P \leqslant p)=$ $\mathscr{P}(P<p)$. Now $I(0)=I(1)=\cdots=I(r)=0$ implies that $P \leqslant(1-\delta)^{r+1}$ and so

$$
F\left((1-\delta)^{r+1}\right) \geqslant(1-\lambda)^{r+1} .
$$

Let $n(\delta)$ be the least integer $n$ such that $\delta(1-\delta)^{n}<1$. For $\delta<\frac{1}{2}, n(\delta)$ is negative while for $\delta \geqslant \frac{1}{2}, n(\delta)$ is 0 . If $P<(1-\delta)^{r+1}$ we have $I(0)=I(1)=\cdots=$ $I(n(\delta)+r)=0$ which means that 


$$
F\left((1-\delta)^{r+1}\right) \leqslant(1-\lambda)^{r+n(\delta)+1} .
$$

Now making the substitution $p=(1-\delta)^{r+1}$ and defining

$$
\alpha=\log (1-\lambda) / \log (1-\delta)
$$

we get

$$
p^{\alpha} \leqslant F(p) \leqslant p^{\alpha}(1-\lambda)^{n(\delta)}
$$

and it is clear that the distribution of $P$ has an infinite mode at 0 or is thin at 0 according to whether $\delta>\lambda$ or $\delta<\lambda$. Since the distribution of $1-P$ is merely the distribution of $P$ with $1-\lambda$ substituted for $\lambda$, we see that there is an infinite mode at 1 or thinness at 1 according to whether $\delta>1-\lambda$ or $\delta<1-\lambda$. Thus the most stable coexistence occurs when $\delta \leqslant \lambda \wedge(1-\lambda)=\frac{1}{2}-\left|\lambda-\frac{1}{2}\right|$ which gives thinness at both 0 and 1 . This means that both species will be found very infrequently at small population sizes. How infrequently depends on the ratios $\log (1-\lambda) / \log (1-\delta)$ and $\log \lambda / \log (1-\delta)$ which repeats our earlier theme that stability increases as $\delta$ decreases.

As $\delta \rightarrow 0$ the distribution of $P$ takes on the familiar normal form $\left(\delta^{-\frac{1}{2}}(P-\lambda)\right.$ is asymptotically normal with mean 0 and variance $\frac{1}{2} \lambda(1-\lambda)$ or $N\left(0, \frac{1}{2} \lambda(1-\lambda)\right)$ ), but even more can be said: the entire stochastic process can be approximated by the Ornstein-Uhlenbeck process as follows.

Theorem 6.1. Let

$$
X_{\delta}(t)=\delta^{-\frac{1}{2}}\left[P_{1}(t / \delta)-\lambda\right]
$$

for $t=0, \delta, 2 \delta, \ldots$ and $X_{\delta}(t+h)=X_{\delta}(t)$ for $h \in[0, \delta)$. If $X_{\delta}(0)$ converges in distribution as $\delta \rightarrow 0$ then the entire process $\left\{X_{\delta}(t), t \geqslant 0\right\}$ converges in distribution to a Markov process $\{X(t), t \geqslant 0\}$ such that the distribution of $X(t)$ given $X(s)$ is $N\left(e^{-(t-s)} X(s), \frac{1}{2} \lambda(1-\lambda)\left(1-e^{-2(t-s)}\right)\right)$.

Remark. The processes $X_{\delta}$ and $X$ have distributions on the space of functions on $[0, \infty)$ that are right continuous and have left limits. This space is given the Skorohod topology (Billingsley, 1968) and the above convergence in distribution is weak convergence of measures on this space. Note that $X$ is an Ornstein-Uhlenbeck process (e.g. Breiman, 1968).

Proof. The process $X_{\delta}$ is a first order autogressive process and I presume that Theorem 6.1 is therefore well known. But if not the theorem is a special case of an obvious minor extension, to processes on $(-\infty, \infty)$, of Turelli's (1977) appendix proposition.

It follows from the theorem that if $\delta$ is small and $P_{1}(0)$ has the approximate stationary distribution $N\left(\lambda, \frac{1}{2} \delta \lambda(1-\lambda)\right)$, then $\left\{P_{1}(t)\right\}$ will be approximately a stationary normal process (the stationary Ornstein-Uhlenbeck process) and $P_{1}(t)$ and $P_{1}(s)$ will have approximate correlation $e^{-\delta|t-s|}$. Thus for the special case considered here we have a very good description of the population fluctuations.

The Ornstein-Uhlenbeck process also arises as an approximate description of population fluctuations about equilibrium when small amounts of temporal environmental variability, or within-individual variability, are added to a deterministic model having a stable equilibrium (May, 1974; Barbour, 1976). 
Coexistence in the lottery model does not involve a stable equilibrium yet the population fluctuations observed are similar to those found when coexistence is explained by a stable equilibrium. Thus a study of population fluctuations alone cannot distinguish an equilibrium explanation of coexistence from the kind of nonequilibrium explanation considered here.

\section{Stochastic Boundedness and Finite Populations}

In previous sections we have learned how environmental fluctuations çan lead to a situation where both species are s.b. persistent and we would like to interpret this as a demonstration that the two species coexist. We now try to justify this conclusion by taking a detailed look at stochastic boundedness and seeing how it applies to the lottery model. Like most analytical models in population ecology the lottery model treats population size as a continuous variable and ignores variability relevant to small populations. Thus we refer to it as an "infinite" population model. Like most stochastic persistence criteria, stochastic boundedness is designed for infinite population models. In order to see that stochastic boundedness is a sensible persistence criterion we try to understand what, if anything, it has to say about "finite" population models in which population size is a discrete variable and which include phenomena like within-individual variability (Chesson, 1978) allowing chance extinction to occur at low densities. Only in this way can we see how to interpret stochastic boundedness in the real world.

\section{Stochastic Boundedness}

Persistence in the sense of stochastic boundedness entails the existence of a positive random variable, with distribution function $G_{i}$, such that

$$
G_{i}(p) \geqslant \mathscr{P}\left(P_{i}(t) \leqslant p\right)
$$

for every $p$. The least possible distribution satisfying (38) is defined by the formula

$$
G_{i}(p)=\sup _{\mathrm{t}} \mathscr{P}\left(P_{i}(t) \leqslant p\right),
$$

at continuity points of the RHS. This distribution will be called the bounding distribution.

Since $G_{i}$ is the distribution of a positive random variable $G_{i}(p) \rightarrow 0$ as $p \rightarrow 0$ which means small population densities have probabilities that are small uniformly in time. It is also helpful to keep in mind that this property of the probabilities imposes a corresponding property on the expected proportion of any set of times having $P_{i}(t) \leqslant p$. In particular letting $\gamma(p, A)=$ proportion of times $t \in A$ such that $P_{i}(t) \leqslant p$ we have

$$
E \gamma(p, A) \leqslant G_{i}(p)
$$

for bounded $A$. When $A$ is unbounded $\gamma(p, A)$ may not be well defined but we can define an asymptotic expected proportion as

$$
E \gamma(p, A) \stackrel{\text { def }}{=} \varlimsup_{t} E \gamma\left(p, A_{t}\right)
$$


where $A_{t}=\{1,2, \ldots, t\} \cap A$. Such asymptotic expected proportions are also bounded by $G_{i}(p)$. Thus expected proportions have the property of being uniformly small for small population densities.

The above observations contain the main intuitive idea behind stochastic boundedness, that is, small populations are seen infrequently. Stochastic boundedness implies a steadiness to population fluctuations. No trends to ever lower population densities are possible and the average frequency of fluctuations to low density does not increase with time.

In conjunction with these qualitative ideas one can use the bounding distribution to indicate the strength of the stochastic boundedness. For example, consider the case of infinite variability in the lottery model with the stationary distribution as the initial distribution. In this case the bounding distribution is the stationary distribution and we know from section 6 , above, that

$$
G_{i}(p)=0\left(p^{\alpha}\right)
$$

for small $p$ where $\alpha=\ln (1-\lambda) / \ln (1-\delta), \lambda$ is mean density and $\delta$ is the death rate. Since $\alpha$ can take on any positive value there is a broad range of possibilities for the rate at which $G_{i}(p) \rightarrow 0$ as $p \rightarrow 0$ with a broad range of interpretations of the idea that small populations have small probabilities of occurring at any given time. In this example a large value of $\alpha$, so that $G_{i}(p)$ converges rapidly to 0 , is a much stronger stochastic boundedness than is found when $\alpha$ is near 0 which means that the bounding distribution has an infinite mode at 0 .

In the above example we had to assume that the initial distribution was the stationary distribution before the bounding distribution was known. However since the process is asymptotically stationary this bounding distribution always describes the behavior of the process in the long term. Since long-term behavior is often the most relevant we are led to define an asymptotic bounding distribution according to the formula

$$
G_{i}^{*}(p)=\varlimsup_{t \rightarrow \infty} \mathscr{P}\left(P_{i}(t) \leqslant p\right) .
$$

The asymptotic bounding distribution will give a better indication of the long-term behavior than $G_{i}(p)$ because it will be less dependent on the initial conditions and will equal the limiting distribution of $P_{i}(t)$ as $t \rightarrow \infty$, when it exists.

If stochastic boundedness is to be a useful criterion for population persistence the alternative situations should describe populations which we naturally regard as not persistent. If a species is not s.b. persistent then there is a positive number $\varepsilon$, an infinite sequence $\left\{t_{n}\right\}$ of times, and a corresponding sequence $\left\{p_{n}\right\}$ of population densities converging to 0 such that

$$
\mathscr{P}\left(P_{i}\left(t_{n}\right) \leqslant p_{n}\right) \geqslant \varepsilon .
$$

As a consequence of (44) we have

$$
E \gamma\left(p,\left\{t_{1}, t_{2}, \ldots\right\}\right) \geqslant \varepsilon
$$

for every positive $p$ so the asymptotic expected proportion of $\left\{t_{1}, t_{2}, \ldots\right\}$ which is spent below any positive density is at least $\varepsilon$. Looking only at $\left\{t_{1}, t_{2}, \ldots\right\}$ one is led to conclude that much time is expected to be spent at very low densities. However this 
conclusion need not hold if one looks at other sets of times. In particular (44) does not imply the existence of a positive $\varepsilon^{\prime}$ such that

$$
\operatorname{E\gamma }(p,\{1,2, \ldots\}) \geqslant \varepsilon^{\prime}
$$

for all $p$, unless

$$
\varlimsup \frac{n}{t_{n}}>0
$$

which requires that the times $t_{n}$ do not become increasingly spread out as time progresses. When (46) does hold the expected proportion of $\{1,2, \ldots, t\}$ spent below any given positive density will approach $\varepsilon^{\prime}$, at least, for some infinite sequence of values of $t$. Thus there will be periods during which large amounts of time are expected to be spent at very low density. This will not be true when (44) but not (46) holds which suggests taking (46) as a minimal definition of a population which does not persist, and taking the strict alternative to this as a definition of a persisting population. However it seems likely that (47) will be satisfied in most instances in practice and so the extra complication of a definition in terms of (46) seems hardly worthwhile.

Expression (46) contains the intuitive notion of spending longer and longer times at lower and lower densities and so either the population is undergoing a general decline or population fluctuations are becoming worse through time. However the population may behave in one of these ways with some probability rather than with any certainty as we shall see below. Spending longer and longer times at lower and lower densities probably means that the population actually becomes extinct due to the effects of finite population size, which are not included in the model. This is discussed in detail below.

In the lottery model we have seen three different circumstances where s.b. persistence does not occur. First there is the situation where $\Delta_{i}<0, \Delta_{j}>0$. Then $\mathscr{P}\left(P_{i}(t) \rightarrow 0\right)=1$ so that $\mathscr{P}\left(P_{i}(t) \leqslant p\right) \rightarrow 1$ for all positive $p$ and also $E \gamma(p, A)=1$ for every unbounded set $A$ and all positive $p$. Since $P_{i}(t)$ declines to 0 it naturally must spend much time at low densities.

Secondly there is the case $\Delta_{1}<0, \Delta_{2}<0$ which can arise when death rates are highly variable and negatively correlated. Then there are $\varepsilon_{1}, \varepsilon_{1}>0$ with $\varepsilon_{1}+\varepsilon_{2}=1$ such that $P_{i}(t) \rightarrow 0$ with probability $\varepsilon_{i}$. It follows that $\mathscr{P}\left(P_{i}(t) \leqslant p\right) \rightarrow \varepsilon_{i}$ and $E \gamma(p, A)=\varepsilon_{i}$ for every unbounded $A$ and positive $p$. In this case only one species actually spends much time at low densities and that species is $i$ with probability $\varepsilon_{i}$.

Finally there is the situation $\Delta_{1}=\Delta_{2}=0$ which we have only analyzed for the case of nonoverlapping generations. Then $\log P_{1}(t) / P_{2}(t)$ undergoes a mean 0 random walk and we have $\mathscr{P}\left(P_{i}(t) \leqslant p\right) \rightarrow \frac{1}{2}, i=1,2$ and $E \gamma(p, A)=\frac{1}{2}$ for every unbounded $A$. The quantities $\mathscr{P}\left(P_{i}(t) \leqslant p\right)$ and $E \gamma(p, A)$ associated with the stochastic boundedness criterion do not reveal obvious differences between this situation and the previous one where $A_{i}<0, i=1,2$. In both the expected amount of time spent at low densities is large but only in the present situation, where $\log P_{1}(t) / P_{2}(t)$ simply undergoes highly unpredictable fluctuations, do both species actually spend much time at low densities. However the distinction between these two cases disappears if one includes the effects of finite population size. This is discussed below where we shall argue that in both cases relatively rapid extinction 
of one species is the correct interpretation with the identity of the species becoming extinct not being predictable a priori.

\section{The Interpretation of Stochastic Boundedness for Finite Populations}

The decision above to regard populations as not persistent because an asymptotic expected proportion of time is spent at arbitrarily low densities emphasizes some artificial features of infinite population models. In these models extinction in finite time is generally impossible (Chesson, 1978) and so we must consider other properties of the model which indicate tendencies toward extinction. This has been the motivation behind stochastic boundedness and the associated bounding distributions and asymptotic expected proportions. The other persistence criteria discussed here, invasibility, convergence to 0 with probability 0 , and convergence in distribution to a positive random variable, are also designed for infinite population models. If such persistence criteria are useful they should give conclusions that are not altered substantially when the model is made more realistic by having population size a discrete variable and including factors like within-individual variability, i.e., these persistence criteria should give useful indications of the behavior of finite versions of the model.

Infinite population models are often available as large population approximations to finite population models. Obtaining an infinite model as a limit of a finite model usually involves letting some parameter that limits total population size, tend to oo (Kurtz 1970, Wang 1975, Chesson 1978, 1981). This parameter which we shall call the "population scale" might be the carrying capacity, the total area of the habitat or, in the lottery model, the total number of territories available. Population density is expressed in units of population scale and the infinite model approximately describes the behavior of population density for large values of the population scale. In Appendix 4 a finite version of the lottery model is defined and it is shown that this finite model does indeed converge to the infinite model discussed in this paper.

Assessing persistence in a finite model is not straightforward. Such models generally have the unhelpful property that eventual extinction occurs with probability 1 . It is for this reason that none of the persistence criteria discussed previously, including stochastic boundedness, apply to finite populations. In the presence of inevitable extinction, attention shifts to the time at which extinction occurs and the nature of population fluctuations before extinction. One might call a population persistent if it has very low probability of becoming extinct on an ecological time scale and, when viewed on an ecological time scale, the population fluctuations are steady in the sense of not showing population crashes of ever increasing severity or a general tendency to decline.

There are a number of difficulties with this definition of persistence in a finite model. First, finite models are usually quite difficult to analyze. Although diffusion approximations can be helpful here (Ludwig, 1976) they are not always desirable. Secondly a number of decisions have to be made. One has to decide what is meant by an ecological time scale. Also one has to decide the relevant population scale since the waiting time for extinction is greatly affected by population scale. For small populations extinction is usually quite rapid but as the population scale is 
increased, and an infinite model is approached, the waiting time converges to infinity because infinite models do not generally permit extinction in finite time (Chesson, 1978). The necessity of these decisions suggests that any theoretical investigations must be qualified by a statement of the relevant ecological time scale and population scale to which they apply. For example in the common situation of a model with parameters that can take different values to represent different situations, one might expect the parameter values that lead to persistence to vary with the time scales and population scales chosen.

Results of Ludwig (1976) and Barbour (1976) suggest a way around some of these problems. Ludwig considers a diffusion model of a finite population growing logistically and affected by both environmental variability and within-individual variability. There are two cases corresponding to s.b. persistence or not in the infinite limit of this finite model. When the infinite model gives s.b. persistence, the expected waiting time for extinction in the finite model is asymptotically some power of the population scale. Otherwise the expected waiting time for extinction is asymptotically equal to $\log K$ or $(\log K)^{2}$ where $K$ is the population scale. Thus the expected waiting time for extinction converges to $\infty$ at a much faster rate when the infinite model gives s.b. persistence than when it does not. An analogous phenomenon to this has been documented by Barbour (1976), for the case of a constant environment, where the waiting time for leaving a neighborhood of equilibrium increases with $K$ at quite different rates depending on the stability of the equilibrium.

When two situations give rise to substantially different rates of increase for the waiting time for extinction, sufficiently large values of $K$ will lead to substantial differences between the actual waiting times for extinction. Thus for population scales above some particular level there will be a large range of values for the time scale on which persistence clearly occurs in one situation and extinction in the other. In other words the conclusions concerning persistence will be quite insensitive to the actual population and time scales chosen. This property will only be of use if the large difference in the waiting times for extinction appears on population and time scales that can be regarded as realistic in the real world. Unfortunately there are little data on this at the present time especially for the cases of main interest here where the environment is stochastic. The numerical results of Ludwig (1976) are unfortunately too limited for the purpose of seeing if large differences in extinction times do appear on realistic population and time scales. The best evidence can be found in a series of papers (Bartlett 1957, Leslie 1958, Leslie and Gower 1958, 1960, Bartlett et al. 1960) for constant environment models where stability or not of the infinite model leads to enormous differences in waiting times for extinction on realistic population and time scales.

As mentioned above the dichotomy in rates of increase of the waiting time for extinction observed by Ludwig corresponded to s.b. persistence or not in the infinite version of the model. There are strong intuitive reasons for expecting that the stochastic boundedness criterion, applied to the infinite version of the model, may quite generally determine a dichotomy in the rates at which waiting times for extinction increase with population scale, $K$, in the finite model. The fact that the finite model converges to the infinite model means that for large values of $K$ the fluctuations in population density in the finite model will remain similar to those in 
the infinite model for long periods of time. Deviations will enter mainly at low densities where extinction may result in the finite model. Stochastic boundedness ensures that a small proportion of time is spent at low densities and the alternative generally means that arbitrarily low densities will be seen with some fixed probability. This dichotomy seems likely to bring about a similar dichotomy in waiting times for extinction and how rapidly they converge to $\infty$ as functions of population scale. This conjecture is the subject of ongoing research but much can be learned by taking the following simplistic approach.

Let $T_{K}$ be the amount of time which must elapse in the infinite model before the population is expected to have spent a time $\tau$ less than or equal to the density $m / K$. The number $m$ is interpreted as an absolute population size in the finite model. Thus $T_{K}$ indicates how long one must wait before expecting to have seen $\tau$ times with absolute population sizes less than $m$. The rate at which $T_{K}$ increases with $K$ is of interest in its own right, but in addition we shall take it as an indication of how quickly the waiting time for extinction in the finite model ought to increase as a function of $K$. For this interpretation we note that the finite and infinite models should behave similarly for absolute densities above $m$ provided $m$ is reasonably large. We also assume that $\tau$ is the amount of time necessary to have spent below the population size $m$ to give a reasonable chance that extinction has occurred in the finite model. The chief justification for taking the behavior of $T_{K}$ as an indication of the behavior of waiting times for extinction is that it works for Ludwig's model; and although Ludwig's model is highly specific the dynamics at low population density are representative of a broad class of situations. However, as with all simplistic treatments of probability there are almost certainly many situations in which the present analysis is misleading.

The quantity $T_{K}$ satisfies the equation $T_{K} E \gamma\left(m / K,\left\{1,2, \ldots, T_{K}\right\}\right)=\tau$ or equivalently

$$
\sum_{u=1}^{T_{K}} F_{u}(m / K)=\tau
$$

where $F_{t}(p)=\mathscr{P}\left(P_{i}(t) \leqslant p\right)$. For a stochastically bounded situation this means $T_{K} G_{i}(m / K) \geqslant \tau$ so that

$$
T_{K} \geqslant \frac{\tau}{G_{i}(m / K)}
$$

and we see that the rate of convergence of $T_{K}$ to $\infty$ is critically dependent on the shape of the bounding distribution at low density. For the lottery model with extreme variation in birth-competition we know that $G_{i}(m / K) \sim(m / K)^{\alpha}$, $\alpha=\ln (1-\lambda) / \ln (1-\delta)$, so that

$$
T_{K}=0\left(K^{\alpha}\right) \text {. }
$$

Thus $T_{K}$ increases as a power law as it does in Ludwig's stochastically bounded cases. The assumptions leading to stochastic boundedness in the general form of the lottery model also imply that $T_{\mathrm{K}}$ increases at least as fast as some positive power of $K$ (see the proof of Theorem 3.2, Appendix 3) but there is no simple formula for $\alpha$.

For the cases where stochastic boundedness does not occur in the lottery model we only know $F_{t}(p)$ when generations are nonoverlapping. Then $\log P_{1}(t) / P_{2}(t)$ is 
asymptotically normal with mean $\log P_{1}(0) / P_{2}(0)+\mu t$ and variance $t \mathscr{V} \log \rho$. For $\mu<0$ and large $t$ this is also the approximate distribution of $\log P_{1}(t)$. Substituting in (48) we obtain

$$
T_{K}=0(\log K) .
$$

In the general case of the lottery model with $\Delta_{1}<0$ and $\Delta_{2}>0$ we know that $\log P_{1}(t)$ is eventually bounded above by a random walk with negative mean and it is not difficult to see that (51) holds there too.

In the case where $\mu=0$ and generations are nonoverlapping the asymptotic normal distribution leads to

$$
T_{K}=0\left((\log K)^{2}\right) .
$$

Thus for the lottery model, in the cases where the information is available, $T_{K}$ converges to $\infty$ at quite different rates depending on whether it is s.b. persistent or not. Indeed if one takes the ratio of $T_{K}$ in any s.b. persistent case, to that in any non s.b. persistent case then this ratio converges to $\infty$ with $K$. However it should also be appreciated that the rate of increase of $T_{K}$ within s.b. persistent cases varies substantially. For example in the case of extreme variability with $\lambda=E P_{1}(t)=\frac{1}{2}$ and $\delta=0.1, T_{K}=0\left(K^{3.32 \cdots}\right)$ while with $\lambda=0.1$ and $\delta=0.5, T_{K}=0\left(K^{0.30 \cdots}\right)$. Moreover in the latter case $T_{K}$ diverges from $\log K$ or $(\log K)^{2}$ at very slow rates so that really very large population scales might be needed before this case of s.b. persistence is clearly distinguishable from cases which are not s.b. persistent. Indeed the situation where $T_{K}$ increases as some small power of $K$ barely deserves to be called persistence. It seems inevitable that there will be a grey zone where $T_{K}$ is in a range which does not allow the model to be classified as giving persistence or not. Thus we conclude that s.b. persistence by itself is not adequate for confidence that a situation is really one of population persistence. Some knowledge of the bounding distribution is necessary also.

In addition to information about $T_{K}$, the bounding distribution indicates the nature of population fluctuations in the infinite model. For large $K$ the fluctuations in the finite model will be similar to those in the infinite model over a long period of time. We know that stochastic boundedness confers some degree of stability on population fluctuations in the infinite model, depending on the bounding distribution. Thus a corresponding degree of stability of population fluctuations is also conferred on the finite model for large $K$. We conclude that stochastic boundedness, together with a suitable bounding distribution and sufficiently large $K$, is capable of ensuring the other part of our requirement for persistence in finite populations viz. population fluctuations are stable over ecological time.

The persistence criteria invasibility and convergence to 0 with probability 0 , are useful steps on the way to proving stochastic boundedness, but they do not imply stochastic boundedness and indeed may permit large amounts of time to be spent at arbitrarily low densities, i.e., relation (46) can be satisfied. Thus persistence according to either of these criteria does not guarantee stable population fluctuations nor long waiting times for extinction with large $K$. Convergence in: distribution to a positive random variable implies stochastic boundedness and the asymptotic bounding distribution is the limiting distribution. Thus persistence may be inferred from the existence of a suitable limiting distribution. The main question 
here is whether convergence in distribution to a positive random variable is superior to stochastic boundedness as a persistence criterion. Knowledge of the existence of a limiting distribution is certainly useful information but it provides no further protection against long periods at low density than stochastic boundedness. Therefore it should not be necessary to prove convergence in distribution to a positive random variable before persistence is concluded.

\section{Discussion}

We have shown that a stochastic environment can be the mechanism of coexistence of competing species. In the lottery model the important features are overlapping generations and variation in birth-competition so that both species have favorable periods, i.e., periods when they are increasing. Section 4 shows mathematically how these features lead to coexistence but the following intuitive explanation helps to indicate the generality of the results.

In general situations with overlapping generations, the adult population may act as a store of the effect of large recruitments, e.g., if a large cohort is recruited during a season, and adult death rates are low, this cohort may dominate the population for several subsequent seasons. Indeed this phenomenon is not uncommon in commercial fisheries. As we shall see below, the potential for storage of the effects of favorable periods leads to an asymmetry in the way in which favorable and unfavorable periods contribute to the mean instantaneous growth rate of a species. This asymmetry is especially important for coexistence in the lottery model where a favorable period for one species is necessarily unfavorable for the other species.

For a species at low density, in the lottery model, periods which are favorable as a result of relatively high birth-competition lead to arbitrarily large instantaneous growth rates. However periods which are unfavorable, because of relatively low birth-competition, must have instantaneous growth rates exceeding $\log \left(1-\delta_{i}\right)$, the instantaneous survivorship of adults, which reflects the capacity to store the effects of previous favorable periods. As a result of this lower bound on growth rates, increasing variation in birth-competition, so that the favorable periods become more favorable and the unfavorable periods less favorable, tends to increase the boundary growth rate (the mean instantaneous growth rate at low density). With high variability the effect of unfavorable periods is small by comparison with that of the favorable periods. This is reflected in the asymptotic expressions $\Delta_{1} \sim E(\log \rho)^{+}, \Delta_{2} \sim E(\log \rho)^{-}$and the observation that each species can persist provided its favorable periods are sufficiently favorable, independently of how unfavorable the unfavorable periods might be. Consequently both species can persist under quite broad conditions when the environment varies.

The above discussion deals with how variation affects mean instantaneous growth rates at low density. Since one species is at high density while the other is at low density and one species gain is the other's loss, there are corresponding implications for high density. This is discussed fully in Chesson and Warner (1981), but the main point is that unfavorable periods make the more important contribution at high density because the gains that can be made by a species at high density are constrained by the other species' death rate. The asymmetry between 
favorable and unfavorable periods, and the reversal of the relative importance of the two for low versus high density, permits coexistence in a variable environment even though it is not possible in a constant environment.

As might the expected the asymmetries between favorable and unfavorable periods are accentuated by low death rates and coexistence is then more likely. On the other hand when generations are nonoverlapping there are no asymmetries in the way favorable and unfavorable periods contribute to the boundary growth rates. Consequently the mechanisms above do not operate in this case and coexistence is impossible.

Coexistence also fails to occur as a result of death rate variation alone. This is easier to understand when it is appreciated that there are definite upper limits on the instantaneous growth rates as functions of adult death rates, but there are no lower limits. For example the instantaneous growth rate of species 1 will be large and negative if $\delta_{1}$ is large and $\delta_{2}$ is small. A large $\delta_{1}$ means that most of the adults die, so that the effect of previous favorable periods is very weak, and a small $\delta_{2}$ means that species 2 gives up very little space so that at low densities of species 1 , little space is being opened up that might allow the expansion of species 1 . With these considerations it is not surprising that death rate variation alone is not beneficial to coexistence. However we have seen situations where death rate variation in combination with variation in birth-competition, is beneficial to coexistence. Moreover in this paper death rates always mean death rates of established adults. Death rates of juveniles contribute to the birth-competition parameters $\beta_{i}^{*}$ and in the real world they may be the chief contributors to variation in birth-competition. Thus according to our model, juvenile death rate variation may be an important contributor to coexistence.

The key factors involved in coexistence in the lottery model, high variability in recruitment rates (birth-competition) and small aduit death rates, could be included in other models. Provided per capita recruitment rates can be high at low population density then there is the potential for asymmetric contributions to the instantaneous growth rate of favorable versus unfavorable periods, and this may benefit coexistence, especially in situations where two species cannot have favorable periods simultaneously. Indeed, it has been shown that environmental variability and overlapping generations continue to promote coexistence in a class of generalizations of the lottery model (Chesson, in prep.; Warner and Chesson, in prep.).

The mechanism of coexistence in the lottery model is stochastic, yet the nature of the coexistence is not dissimilar to that found commonly in models having a deterministic mechanism in a stochastic environment (e.g., Turelli and Gillespie, 1980). In both, species densities converge in distribution to positive random variables. Moreover in the lottery model with extreme variation. in birthcompetition, but small adult death rates, the adult population will fluctuate very little and in some cases can be described approximately by the same stochastic process (the Ornstein-Uhlenbeck process) that is used to approximate fluctuations about equilibrium in a stable deterministic model to which a small amount of environmental variability, or within-individual variability, has been added. Indeed a stochastic mechanism relying on large environmental fluctuations need not lead to large population fluctuations. 
To judge whether the nature of the population fluctuations is indicative of persisting species we have used the stochastic boundedness criterion. This criterion requires that the probability of observing a population below any given density, should converge to 0 with density, uniformly in time. Consequently it places restrictions on the expected frequency of fluctuations to low population levels. Given that fluctuations in the environment will continually perturb population densities, it is to be expected that any nominated population density, no matter how small, will eventually be seen. Indeed this is the usual case in stochastic population models and is not an unreasonable postulate about the real world. Thus a reasonable persistence criterion cannot hope to do better than place restrictions on the frequencies with which such events occur.

In an infinite population model, s.b. persistence together with a bounding distribution which converges rapidly to 0 as densities approach 0 , seems to provide a suitable guarantee of infrequent low populations and long waiting times for extinction in finite versions of the model. We have only sought detailed information on the bounding distribution for the version of the model with infinite variation in birth-competition, and constant but equal death rates. For this case the bounding distribution converges rapidly to 0 with population density, provided adult death rates are small. There are strong indications that this may be true generally for the lottery model giving another reason for believing that small adult death rates are important for coexistence in the lottery competition model.

\section{Appendix I}

Proofs of Results from Section 3

Proof of Lemma 3.2. Let $c>0$ be such that $E \log \{1+\Gamma(0) \vee \Gamma(c)\}<0$. To show that $P_{1}(t) \in(0, c)$ i.o. implies $P_{1}(t) \rightarrow 0$, a.s. we define

$$
\begin{aligned}
A & =\left\{P_{1}(t) \in(0, c) \text { i.o. }\right\} \\
B & =\left\{P_{1}(t) \rightarrow 0 \text { as } t \rightarrow \infty\right\} \\
C & =\left\{P_{1}(t) \in[c, 1) \text { i.o. }\right\} .
\end{aligned}
$$

Let $\tau_{n}$ be the $n$th time that $P_{1}$ enters $(0, c)$ from $[c, 1)$. Define $\tau_{n}=\infty$ if there is no such time. Now

$$
\left\{\tau_{n+1}<\infty\right\} \subseteq C_{n} \stackrel{\text { def }}{=}\left\{\prod_{s=\tau_{n}}^{\tau_{n}+t}[1+\Gamma(s, 0) \vee \Gamma(s, c)]>1 \text { for some } t \geqslant 0\right\} \cap\left\{\tau_{n}<\infty\right\}
$$

and, from the theory of random walks (Feller, 1971) it is clear that

$$
p=\mathscr{P}\left(\prod_{s=0}^{t}\{1+\Gamma(s, 0) \vee \Gamma(s, c)\}>1 \text { for some } t \geqslant 0\right)<1 .
$$

The $\tau_{n}$ 's are stopping times for the process (Revuz, 1975) and so

$$
\mathscr{P}\left(C_{n} \mid H_{\tau_{n}}\right) \leqslant p 1_{\left[\tau_{n}<\infty\right)}
$$

where $H_{\tau_{m}}$ is the $\sigma$-field associated with $\tau_{n}$. It follows that

$$
\mathscr{P}\left(\tau_{n+1}<\infty \mid H_{\tau_{n}}\right) \leqslant p 1_{\left.i_{n}<\infty\right\}} .
$$

Hence $\mathscr{P}\left(\tau_{n+1}<\infty\right) \leqslant p \mathscr{P}\left(\tau_{n}<\infty\right)$ and so $\mathscr{P}\left(\tau_{n}<\infty\right) \rightarrow 0$ as $n \rightarrow \infty$. Finally $\left\{\tau_{n}<\infty\right\} \downarrow C$ giving $\mathscr{P}(C)=0$. Thus, on $A$, there is a time $\tau$ such that $P_{1}(t) \in(0, c)$ for $t \geqslant \tau$, a.s. 
Now, on $A$

$$
P_{1}(t+\tau) / P_{1}(\tau) \leqslant \prod_{s=\tau}^{t+\tau}\{1+\Gamma(s, 0) \vee \Gamma(s, c)\} .
$$

From the law of large numbers RHS $\rightarrow 0$ as $t \rightarrow \infty$, a.s. Hence $P_{1}(t) \rightarrow 0$ as $t \rightarrow \infty$, on $A$, a.s., which is the required result.

Proof of Lemma 3.3. For the case where $\rho$ is not a.s. equal to 1 , we must show that $P_{1}(t)$ will be found outside $A=[\varepsilon, 1-\varepsilon]$ i.o., a.s. for every $\varepsilon>0$. First of all note that

$$
\varepsilon /\left\{\beta_{1}(1-\varepsilon)+\beta_{2} \varepsilon\right\} \leqslant(1-p) /\left\{\beta_{1} p+\beta_{2}(1-p)\right\} \leqslant(1-\varepsilon) /\left\{\beta_{1} \varepsilon+\beta_{2}(1-\varepsilon)\right\} .
$$

For definiteness assume $\mathscr{P}(\rho>1)>0$, then, defining $d=\Gamma(1-\varepsilon)$ we have $\mathscr{P}(d>0)>0$ and hence a positive number $\gamma$ such that $\mathscr{P}(d>\gamma)>0$. For given $\varepsilon>0$ there is a positive integer $r$ such that $\varepsilon(1+\gamma)^{r}>1-\varepsilon$. Note that $P_{1}(t) \in A, d(t), \ldots, d(t+r-1)>\gamma$, implies $P_{1}(t+r) \notin A$. Define $\tau_{n}=n$th time that $P_{1}(t) \in A . \tau_{n}$ is a stopping time for $P_{1}$. Now $\left\{P_{1}\right.$ leaves $A$ after $\left.\tau_{n}\right\} \supsetneq\{$ there is an $s \geqslant 0$ such that $\left.d\left(\tau_{n}+s\right), \ldots, d\left(\tau_{n}+s+r-1\right)>\gamma\right\}$. Since the sequence $d\left(\tau_{n}\right), d\left(\tau_{n}+1\right), \ldots$ has the same distribution as the sequence $d(0), d(1), \ldots$, this latter event has probability 1 . Hence $P_{1}$ must always leave $A$ eventually, a.s. which means $\mathscr{P}\left(P_{1}(t) \notin A\right.$ i.o. $)=1$.

Proof of Lemma 3.4. To show that $\mathscr{P}\left(P_{1}(t) \in(1-\varepsilon, 1)\right.$ i.o. $)=1$ for $\Delta_{1}>0$ and $\varepsilon>0$, we note that $\mathscr{P}(\rho>1)>0$. Also the proof of Theorem 3.1 shows that there is a $c>0$ for which $P_{1}(t) \in[c, 1)$ i.o., a.s.

Let $\tau_{n}$ be the $n$th $t$ for which $P_{1}(t) \in[c, 1)$. Define $\varepsilon^{\prime}=\varepsilon \wedge c$ and define $d$ and $\gamma$ as in (3.3) with $\varepsilon^{\prime}$ replacing $\varepsilon$ in (3.3). Now $d\left(\tau_{n}\right), n=1,2, \ldots$, is an i.i.d. sequence, and by the law of large numbers for ergodic stationary processes

$$
n^{-1} \sum_{j=1}^{n} 1_{\left\{d\left(\tau_{j+1}\right)>\gamma, l=0, \ldots, r-1\right\}}
$$

converges a.s., as $n \rightarrow \infty$, to

$$
\mathscr{P}\left(d\left(\tau_{n+l}\right)>\gamma, l=0, \ldots, r-1\right)>0
$$

Also,

$$
\left\{d\left(\tau_{n+l}\right)>\gamma, l=0, \ldots, r-1\right\} \subseteq\left\{P_{1}(t) \in[c, 1) \text { for } t=\tau_{n}+1, \ldots, \tau_{n}+r \text { and } P_{1}\left(\tau_{m}+r\right)>1-\varepsilon\right\}
$$

from which it is clear that this latter event occurs infinitely often. It follows that $P_{1}(t) \in(1-\varepsilon, 1)$ i.o., a.s.

\section{Appendix 2}

\section{Inequalities for Boundary Growth Rates}

For the inequalities $(14 a, b)$ we have

$$
\begin{aligned}
\Delta_{1} & =E \log \left\{\delta_{1} \rho+1-\delta_{1}\right\} \\
& >E\left(\log \left\{\delta_{1} \rho+1-\delta_{1}\right\}\right)^{+}+E \log \left(1-\delta_{1}\right) 1_{\{\rho<1\}} .
\end{aligned}
$$

Since $\log$ is concave

$$
\log \left\{\delta_{1} \rho+1-\delta_{1}\right\}>\delta_{1} \log \rho+\left(1-\delta_{1}\right) \log 1
$$

and so we conclude

$$
\Delta_{1}>E \delta_{1}(\log \rho)^{+}+E \log \left(1-\delta_{1}\right) 1_{\{\rho<1\}}
$$

which is $(14 a)$; inequality (14b) is analogous. From (A1) we conclude also that

$$
\begin{aligned}
\Delta_{1} & >E(\log \rho)^{+}+E \log \delta_{1} 1_{\{\rho>1\}}+E \log \left(1-\delta_{1}\right) 1_{\{\rho<1\}} \\
& >E(\log \rho)^{+}+E \log \delta_{1}\left(1-\delta_{1}\right) .
\end{aligned}
$$


It is easy to see that $E(\log \rho)^{+}>\Delta_{1}$ and so $E \log \delta_{1}\left(1-\delta_{1}\right)$ is an absolute bound on $\left|E(\log \rho)^{+}-\Delta_{1}\right|$ depending only on the distribution of $\delta_{1}$.

Approximations to $\triangle_{1}$ for small $\delta_{1}$ are obtained in Chesson and Warner (1981) where it is shown that $x^{-1} \log \left\{1+x \delta_{1}(\rho-1)\right\} \uparrow E \delta_{1}(\rho-1)$ as $x \downarrow$. It follows that

$$
\Delta_{1}<E \delta_{1}(\rho-1) \text {. }
$$

\section{Appendix 3}

\section{Proofs of Stochastic Boundedness}

Proof of Theorem 5.1. To prove that both species are s.b. persistent whenever $E\left(1-\delta_{i}\right)^{-\varepsilon}<\infty$ and $\mathscr{E}$ is an i.i.d. process, we use an adaptation of Norman's (1975) proof of tightness of gene frequency distributions in a genetic model.

Species 1 will be s.b. persistent if for some $\varepsilon>0$ there is a finite constant $M$ such that $E P_{1}^{-\varepsilon}(t) \leqslant M$ because then $\mathscr{P}\left(P_{1}(t)<x\right) \leqslant x^{\varepsilon} M$ and this implies that $\inf _{x>0} \sup _{t} \mathscr{P}\left(P_{1}(t)<x\right)=0$.

Define $Y_{c}=[1+\Gamma(0) \wedge \Gamma(c)]$. For $P_{1}(t) \leqslant c$ we have $P_{1}(t+1) / P_{1}(t) \geqslant Y_{c}$ and therefore

$$
E\left[\left\{P_{1}(t+1) / P_{1}(t)\right\}^{-\varepsilon} \mid P_{1}(t)\right] \leqslant E Y_{c}^{-\varepsilon}
$$

for $P_{1}(t) \leqslant c$. Hence

$$
E\left[P_{1}^{-\varepsilon}(t+1) \mid P_{1}(t)\right] \leqslant P_{1}^{-\varepsilon}(t) E Y_{c}^{-\varepsilon}
$$

for $P_{1}(t) \leqslant c$, while $(27)$ implies that

$$
E\left[P_{1}^{-\varepsilon}(t+1) \mid P_{1}(t)\right] \leqslant c^{-\varepsilon} K
$$

for $P_{1}(t) \geqslant c$. Combining (A6) and (A7) we conclude

$$
E P_{1}^{-\varepsilon}(t+1) \leqslant E P_{1}^{-\varepsilon}(t) E Y_{c}^{-\varepsilon}+c^{-\varepsilon} K
$$

and this inequality holds for all positive $c$ and any $\varepsilon$ sufficiently small.

Now $Y_{c} \geqslant\left(1-\delta_{i}\right)$ and so $E Y_{c}^{-\varepsilon}<\infty$ for $\varepsilon$ sufficiently small. Also $\varepsilon^{-1}\left(Y_{c}^{-\varepsilon}-1\right) \downarrow-\log Y_{c}$ as $\varepsilon \downarrow 0$ and so from the monotone convergence theorem we have $E \varepsilon^{-1}\left(Y_{c}^{-\varepsilon}-1\right) \rightarrow-E \log Y_{c}$ as $\varepsilon \rightarrow 0$. By relation (9), $E \log Y_{c}>0$, for $c$ sufficiently small, giving

$$
E Y_{c}^{-\varepsilon}<1,
$$

for $\varepsilon$ sufficiently small. Combining this with (A8) we obtain

$$
E P_{1}^{-\varepsilon}(t) \leqslant E P_{1}^{-\varepsilon}(0)+c^{-\varepsilon} K /\left(1-E Y_{c}^{-\varepsilon}\right) .
$$

Thus species 1 will be s.b. persistent whenever $E P_{1}^{-\varepsilon}(0)<\infty$. However this condition is not necessary because (A10) continues to hold when the expectations are conditional on $P_{1}(0)$. Thus we have

$$
\inf _{x>0} \sup \mathscr{P}\left(P_{1}(t)<x \mid P_{1}(0)\right)=0 .
$$

The bounded convergence theorem gives

$$
\inf _{x>0} E \sup _{t} \not P\left(P_{1}(t)<x \mid P_{1}(0)\right)=0
$$

and the required s.b. persistence now follows from the general inequality $E \sup _{t} X_{t} \geqslant \sup _{t} E X_{t}$.

By interchange of species names this also shows that species 2 is s.b. persistent.

Proof of Theorem 5.2. To prove that $P_{i}(t)$ converges to a distribution on $(0,1)$ we again use a modification of a proof by Norman (1975).

Since $A_{1}$ and $A_{2}$ are both positive it follows that $\mathscr{P}(\rho<1)$ and $\mathscr{P}(\rho>1)$ are both positive. Lemma 3.4

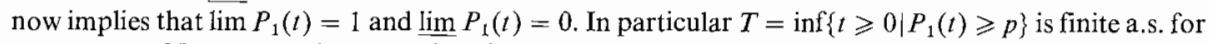
every $p<1$. Note that $T$ is a stopping time (Revuz, 1975).

Let $U$ be the transition operator for the Markov chain $\left\{P_{1}(t)\right\}$, i.e. $U f(p)=E\left[f\left(P_{1}(t)\right) \mid P_{1}(t)=p\right]$ for bounded measurable $f$ on $(0,1)$. From equation (3) it is easily seen that $P_{1}(t+1)$ is an increasing 
function of $P_{1}(t)$, conditional on $\mathscr{E}$. It follows that $U$ maps nondecreasing functions into nondecreasing functions.

For nonnegative, nondecreasing $f$ we have

$$
E f\left(P_{1}(t)\right) \geqslant E\left[f\left(P_{1}(t)\right) ; T \leqslant t\right]
$$

and using the strong Markov property (Revuz, 1975) the RHS is equal to

$$
E\left[U^{t-T} f\left(P_{1}(T)\right) ; T \leqslant t\right] \geqslant E\left[U^{t-T} f(p) ; T \leqslant t\right] .
$$

Applying Fatou's lemma we have

$$
\varliminf_{t \rightarrow \infty} E f\left(P_{\perp}(t)\right) \geqslant E \varliminf_{t \rightarrow \infty} U^{t-T} f(p) 1_{\{T \leqslant t\}}=\varliminf_{t \rightarrow \infty} U^{t} f(p) .
$$

Since this holds for all $p<1$, and the RHS is nondecreasing in $p$, it follows that

$$
\varliminf_{t \rightarrow \infty} E f\left(P_{1}(t)\right) \geqslant U^{\infty} f \stackrel{\text { der }}{=} \lim _{p \rightarrow 1} \varliminf_{i \rightarrow \infty} U^{\prime} f(p) .
$$

On the other hand if $|f(p)| \leqslant M<\infty$, for all $p$, we have

$$
\begin{aligned}
E f\left(P_{1}(t)\right) & =E U^{u} f\left(P_{1}(t-u)\right) \\
& \leqslant E\left[U^{u} f\left(P_{1}(t-u)\right) ; P_{1}(t-u) \leqslant p\right]+E\left[U^{u} f\left(P_{1}(t-u)\right) ; P_{1}(t-u)>p\right] \\
& \leqslant U^{u} f(p)+M \mathscr{P}\left(P_{1}(t-u)>p\right) .
\end{aligned}
$$

Hence

$$
\overline{\lim } E f\left(P_{1}(t)\right) \leqslant U^{u} f(p)+M \sup _{t} \mathscr{P}\left(P_{1}(t)>p\right) .
$$

Now the LHS is independent of $u$ and $p$ while the second term on the RHS $\rightarrow 0$ as $p \rightarrow 1$, by Theorem 5.1. Thus

$$
\varlimsup-\overline{\lim } E f\left(P_{1}(t)\right) \leqslant U^{\infty} f
$$

and therefore

$$
\lim _{t \rightarrow \infty} E f\left(P_{1}(t)\right)=U^{\infty} f
$$

for all bounded nondecreasing $f$, and the limit is independent of the distribution of $P_{1}(0)$. It follows that $P_{1}(t)$ converges in distribution to a random variable $P$ on $[0,1]$. However the s.b. persistence of Theorem 5.1 is equivalent to tightness of $P_{1}(t)$ on $(0,1)$ and so it follows that the distribution of $P$ is concentrated on $(0,1)$. Moreover this distribution is independent of the distribution of $P_{1}(0)$.

\section{Appendix 4}

\section{The Finite Model}

It has been argued in the text that s.b. persistence for an infinite model implies a suitable persistence for large population scales in a finite model converging to the infinite model. We must now define the finite version of the lottery model and show that it does indeed coverge to the infinite version so that the discussion in the text is applicable.

For simplicity in presentation we will assume that $c_{1}=c_{2}$, i.e. the allocation of space to juveniles is purely random, and in setting up the model all statements are conditional on the environment process $\mathscr{E}$. We let $K=$ the number of suitable homesites available to the two species - it is the population scale. We need some definitions:

1. $N_{i}(t)=$ number of adults of species $i$ at time $t$ and $P_{i}(t)=N_{i}(t) / K$.

2. $S_{i}(t+1)=$ number of adults of species $i$ at time $t$ that are still living at time $t+1$.

3. $L_{i}(t+1)=$ number of larvae of species $i$ seeking homes during $(t, t+1]$.

4. $R_{i}(t+1)=$ number of larvae of species $i$ finding homes during $(t, t+1]$. 
The fundamental equations governing the system are now

$$
N_{i}(t+1)=S_{i}(t+1)+R_{i}(t+1),
$$

$i=1,2$. We do not insist that $N_{1}(t)+N_{2}(t)=K$, i.e. some space may remain empty, however our assumptions will entail that $N_{1}(t)+N_{2}(t)=K$ has high probability.

The process $\mathbf{N}=\left\{\left(N_{1}(t), N_{2}(t)\right), t=0,1, \ldots\right\}$ is a Markov process with transition probabilities defined as follows. Given $\mathbf{N}(t)$, the variables $S_{i}(t+1), i=1,2$, are independent binomial random variables with $n$-parameters $N_{i}(t)$ and $p$-parameters $1-\delta_{i}(t)$. That is, given $\mathbf{N}(t)$ and $\mathscr{E}$ the deaths of different adults are independent events.

The number of larvae $L_{i}(t)$ has the representation

$$
L_{i}(t)=\sum_{j=1}^{N_{i}(t)} M_{i j}(t)
$$

where the random variables $M_{i j}(t), i=1,2, j=1, \ldots, N_{i}(t)$ are conditionally independent given $\mathbf{N}(t)$, $E M_{i j}(t)=\beta_{i}(t)$ and the distribution of $M_{i j}(t)$ does not depend on $t, j$ or $K . M_{i j}(t)$ represents the number of offspring during $(t, t+1]$ from the $j$ th adult of species $i$ living at time $t$.

Finally if $K-S_{1}(t+1)-S_{2}(t+1) \geqslant L_{1}(t+1)+L_{2}(t+1)$ then $R_{i}(t+1)=L_{i}(t+1), i=1,2$. Otherwise, given $S_{i}(t+1), L_{i}(t+1), i=1,2, R_{i}(t+1)$ is hypergeometric:

$$
\mathscr{P}\left(R_{i}(t+1)=r \mid S_{j}(t+1), L_{j}(t+1), j=1,2\right)=\frac{\left(\begin{array}{c}
L_{i}(t+1) \\
r
\end{array}\right)\left(\begin{array}{c}
L_{j}(t+1) \\
K-S_{1}(t+1)-S_{2}(t+1)-r
\end{array}\right)}{\left(\begin{array}{c}
L_{1}(t+1)+L_{2}(t+1) \\
K-S_{1}(t+1)-S_{2}(t+1)
\end{array}\right)}
$$

and $R_{1}(t+1)+R_{2}(t+1)=K-S_{1}(t+1)-S_{2}(t+1)$ so that all available space is used up.

We assume that $\beta_{i}(t)>\delta_{i}(t), i=1,2$. When this is so it is easy to see that the transition probabilities have the property that

$$
S_{i}(t+1) / K^{P} p_{i}\left[1-\delta_{i}(t)\right]
$$

$i=1,2$, if $K \rightarrow \infty$ and $P_{i}(t) \rightarrow p_{i}$; and

$$
R_{i}(t+1) / \underset{\text { P }}{\rightarrow}\left[\delta_{1}(t) p_{1}+\delta_{2}(t) p_{2}\right] \beta_{i}(t) p_{i} /\left[p_{1} \beta_{1}(t)+p_{2} \beta_{2}(t)\right],
$$

provided $p_{1}+p_{2}=1$. This means that the conditional distribution of $P_{i}(t+1)$ given $P_{i}(t), i=1,2$, converges on the relationship specified by equation (3). If we also assume that the distribution of $\left(P_{1}(0), P_{2}(0)\right)$ converges as $K \rightarrow \infty$ to a distribution where $P_{1}(0)+P_{2}(0)=1$ and $P_{i}(0)>0$ a.s., then we can prove the following.

Theorem A1. As $K \rightarrow \infty$ the stochastic process $\left\{P_{1}(t), t=0,1, \ldots\right\}$ converges in distribution to the process given by equation (3).

The proof is based on the following lemma where the symbol " $\Rightarrow$ " denotes weak convergence (Billingsley, 1968).

Lemma A2. Let $\pi_{K}$ be a probability measure on the Borel sets $\left(\mathscr{B}^{m}\right)$ in $R^{m}$, for $K=1,2, \ldots$ Let $\gamma_{K}(\cdot, \mathbf{x})$ be a probability measure on $\mathscr{B}^{k}$ for every $\mathbf{x} \in R^{k}$ such that $\gamma_{K}(B, \mathbf{x})$ is measurable in $\mathbf{x}$ for every $B \in \mathscr{B}^{k}$. Suppose that $\pi_{K} \Rightarrow \pi$ as $K \rightarrow \infty$ and $\gamma_{K}\left(\cdot, \mathbf{x}_{K}\right) \Rightarrow \gamma(\cdot, \mathbf{x})$ whenever $\mathbf{x}_{K} \rightarrow \mathbf{x}$ as $K \rightarrow \infty$, then the probability measure

$$
\begin{aligned}
\mu_{\mathbf{K}}\left(B_{1} \times B_{2}\right) & =\int_{\mathbf{B}_{1}} \gamma_{K}\left(B_{2}, \mathbf{x}\right) d \pi_{\mathbf{K}}(\mathbf{x}) \\
\mu\left(B_{1} \times B_{2}\right) & =\int_{B_{1}} \gamma\left(B_{2}, \mathbf{x}\right) d \pi(\mathbf{x}) .
\end{aligned}
$$

Proof. Let $f_{1}$ and $f_{2}$ be bounded continuous functions on $R^{m}$ and $R^{k}$ respectively. Since $\pi_{\mathrm{K}} \Rightarrow \pi$ there is a sequence of random variables $\mathbf{X}_{K}$ with distribution $\pi_{K}$ concerning everywhere to a random variable $\mathbf{X}$ 
with distribution $\pi$ (Billingsley, 1971). It follows that

$$
f_{1}\left(\mathbf{X}_{K}\right) \int f_{2}(\mathbf{y}) \gamma_{K}\left(d \mathbf{y}, \mathbf{X}_{K}\right)
$$

converges everywhere to

$$
f_{1}(\mathbf{X}) \int f_{2}(\mathbf{y}) \gamma(d \mathbf{y}, \mathbf{X})
$$

Applying the dominated convergence theorem we see that

$$
E f_{1}\left(\mathbf{X}_{K}\right) \int f_{2}(\mathbf{y}) \gamma_{K}\left(d \mathbf{y}, \mathbf{X}_{K}\right) \rightarrow E f_{1}(\mathbf{X}) \int f_{2}(\mathbf{y}) \gamma(d \mathbf{y}, \mathbf{X}) .
$$

The LHS and RHS are respectively

$$
\int f_{1}(\mathbf{x}) f_{2}(\mathbf{y}) d \mu_{K}(\mathbf{x}, \mathbf{y}) \quad \text { and } \quad \int f_{1}(\mathbf{x}) f_{2}(\mathbf{y}) d \mu(\mathbf{x}, \mathbf{y})
$$

which proves the lemma.

To prove the theorem we identify $\pi_{K}$ with the distribution of $\left\{\left(P_{1}(s), P_{2}(s)\right), s=0,1, \ldots, t\right\}$ and $\gamma_{K}$ with the conditional distribution of $\left(P_{1}(t+1), P_{2}(t+1)\right)$ given $\left\{\left(P_{1}(s), P_{2}(s)\right), s=0,1, \ldots, t\right\}$. The measure $\gamma$ is the conditional probability measure which makes $P_{1}(t+1)$ satisfy equation (3) with probability 1 whenever $P_{1}(t)+P_{2}(t)=1$. When $P_{1}(t)+P_{2}(t)<1, \gamma$ specifies a slightly different relationship which need not concern us.

The proof is by induction on $t$. If $\left\{\left(P_{1}(s), P_{2}(s), s=0,1, \ldots, t\right\}\right.$ converges in distribution to a process satisfying equation (3) with $P_{1}(t)+P_{2}(t)=1$, then from Lemma A2 and relations (A12) and (A13) it follows that $\left\{\left(P_{1}(s), P_{2}(s), s=0,1, \ldots, t+1\right\}\right.$ also converges in distribution to a process satisfying (A12) and (A13). Since the induction hypothesis is true for $t=0$ we have demonstrated that the finite dimensional distributions of $\left\{\left(P_{1}(t), P_{2}(t)\right), t=0,1, \ldots\right\}$ converge to those of a process satisfying (3). From Billingsley (1968, page 22 ) this is sufficient to imply weak convergence of the infinite dimensional distribution on $R^{\infty}$ with countable product topology.

The proof given is actually for the conditional distribution of $\left\{\left(P_{1}(t), P_{2}(t)\right)\right\}$ given $\mathscr{E}$, but since $\mathscr{E}$ does not change with $K$ this is sufficient to prove the result unconditionally also.

Acknowledgements. I am indebted to Michael Turelli for his continued help and encouragement. Comments on the manuscript by Michael Turelli and Alan Hastings, and ongoing discussions with Robert Warner, have been very valuable. I am grateful for the voluntary labor of Kim McDaniel who typed the original version of the manuscript. This work was supported by NSF grant \# DEB 79-22131.

\section{References}

Armstrong, R. A., McGehee, R.: Coexistence of species competing for shared resources. Theoret. Population Biology 9, 317-328 (1976)

Barbour, A. D.: Quasi-stationary distributions in Markov population processes. Adv. Appl. Probab. 8, $296-314(1976)$

Bartlett, M. S. : On theoretical models for competitive and predatory biological systems. Biometrika $\mathbf{4 4}$, $27-42(1957)$

Bartlett, M. S., Gower, J. C., Leslie, P. H.: A comparison of theoretical and empirical results for some stochastic population models. Biometrika 47, 1-11 (1960)

Beddington, J. R., Free, C. A., Lawton, J. H.: Concepts of stability and resilience in predator-prey models. J. Anim. Ecol. 45, 791-816 (1976)

Billingsley, P.: Convergence of probability measures. New York: Wiley 1968

Billingsley, P.: Weak convergence of measures. Philadelphia: SIAM 1971

Botkin, D. B., Sobel, M. J.: Stability in time-varying ecosystems. Am. Nat. 109, 625-646 (1975) 
Breiman, L.: Probability. Menlo Park, CA: Addison-Wesley 1968

Chesson, P. L.: Predator-prey theory and variability. Ann. Rev. Ecol. Syst. 9, 323-347 (1978)

Chesson, P. L.: Models for spatially distributed populations: The effect of within-patch variability. Theoret. Population Biology 19, 288-325 (1981)

Chesson, P. L., Warner, R. R.: Environmental variability promotes coexistence in lottery competitive systems. Am. Nat. 117, $923-943$ (1981)

Cushing, J. M.: Two species competition in a periodic environment. J. Math. Biol. 10, 385-400 (1980)

de Mottoni, P., Schiaffino, A.: Competition systems with periodic coefficients: A geometric approach. J. Math. Biol. 11, 319-335 (1981)

Feller, W.: An introduction to probability theory and its applications, Vol. II, second edition. New York: Wiley 1971

Goh, B. S.: Stability, vulnerability, and persistence of complex ecosystems. Ecological Modelling 1, $105-116(1975)$

Goh, B. S.: Nonvulnerability of ecosystems in unpredictable environments. Theoret. Population Biology 10, 83-95 (1976)

Heyde, C. C.: On the central limit theorem for stationary processes. Z. Wahrscheinlichkeitstheorie 30 , $315-320(1974)$

Holling, C. S.: Resilience and stability of ecological systems. Ann. Rev. Ecol. Syst. 4, 1-23 (1973)

Ibragimov, I. A.: A note on the central limit theorem for dependent random variables. Theoret. Prob. Appl. 20, $135-141$ (1975)

Karlin, S., Liberman, U.: Random temporal variation in selection intensities: Case of large population size - I. Theoret. Population Biology 6, 355-382 (1974)

Koch, A. L.: Coexistence resulting from an alternation of density independent and density dependent growth. J. Theoret. Biol. 44, $373-386$ (1974)

Kurtz, T. G.: Solutions of ordinary differential equations as limits of pure jump Markov processes. J. Appl. Probab. 7, 49-58 (1970)

Leigh, E. G.: Population fluctuations, community stability, and environmental variability. In: (M. L. Cody, J. M. Diamond, eds.) Ecology and evolution of communities, pp. 51-73. Cambridge MA: Harvard University Press 1975

Leslie, P. H.: A stochastic model for studying the properties of certain biological systems by numerical methods. Biometrika 45, 16-31 (1958)

Leslie, P. H., Gower, J. C.: The properties of a stochastic model for two competing species. Biometrika 45, 316-330 (1958)

Leslie, P. H., Gower, J. G. : The properties of a stochastic model for the predator-prey type of interaction between two species. Biometrika 47, $219-234$ (1960)

Levins, R.: Coexistence in a variable environment. Am. Nat. 114, 765-783 (1979)

Lewontin, R. C.: The meaning of stability. In: Diversity and stability in ecological systems. Brookhaven Symposium in Biology No. 22, 13-24 (1969)

Ludwig, D. : A singular perturbation problem in the theory of population extinction. Soc. Ind. Appl. Math.-Am. Math. Soc. Proc. 10, 87-104 (1976)

May, R. M. : Stability and complexity in model ecosystems, second edition. Princeton, N. J.: Princeton Univ. Press 1974

Norman, F.: An ergodic theorem for evolution in a random environment. J. Appl. Prob. 12, $661-672$ (1975)

Revuz, D.: Markov chains. Amsterdam: North-Holland Publication Co. 1975

Sale, P. F.: Maintenance of high diversity in coral reef fish communities. Am. Nat. 111, 337-359 (1977)

Stewart, F. M., Levin, B. R.: Partitioning of resources and the outcome of interspecific competition: A model and some general considerations. Am. Nat. 107, 171-198 (1973)

Turelli, M.: Random environments and stochastic calculus. Theoret. Population Biology 12, $140-178$ (1977)

Turelli, M. : A reexamination of stability in randomly fluctuating versus deterministic environments. Theoret. Population Biology 13, 244-267 (1978a)

Turelli, M.: Does environmental variability limit niche overlap? Proc. Natl. Acad. Sci. USA 75, $5065-5089(1978 \mathrm{~b})$

Turelli, M.: Niche overlap and invasion of competitors in random environments I. Models without demographic stochasticity. Theoret. Population Biology 20, 1-56 (1981) 
Turelli, M., Gillespie, J. H.: Conditions for the existence of stationary densities for some two dimensional diffusion processes with applications in population biology. Theoret. Population Biology 17, 167-189 (1980)

Wang, F. J. S.: Limit theorems for age and density dependent stochastic population models. J. Math. Biol. 2, $373-400$ (1975)

Received November 14, 1981/Revised January 25, 1982 